\title{
GREEN PRODUCT DIFFUSION: THE IMPACTS OF ASYMMETRIC RETAILERS' STRATEGIC PRODUCT DECISIONS
}

\author{
XiaOxi Zhu ${ }^{1,2}$ AND GuANGdong $\mathrm{WU}^{3, *}$
}

\begin{abstract}
With the continuous deterioration of the environment and the improvement of consumer green awareness, more and more producers began to launch green products. For example, many automobile companies began to produce new energy vehicles. However, whether a new product can be successfully introduced to the market depends not only on the product's quality improvement, but also on its sales channels. In this paper, we model a supply chain composed of a manufacturer and two asymmetric retailers to analyze how the retailers' strategic decisions affect the introduction of a newer green product. Backward induction is adopted to survey the dynamic decisions of the supply chain members. Given the leading retailer's product choice, the follower-up retailer's product choices and decision optimums are defined by specific thresholds of consumer green valuation and production costs. Results show that the follower-up retailer would make completely different responses within a same threshold range when the leading retailer takes different product decisions. In other words, even if the leading retailer chooses green new products, the follower will not necessarily imitate the choice of green products, and it could be more advantageous to choose the old generation products (for price competition). Furthermore, results show that green product introduction does not necessarily bring Pareto improvement to both the two retailers. Finally, we derive the specific intervals in which green products can be successfully introduced into the market. Our modelling work and results provide instructive managerial insights on green product introduction in a retailer led supply chain.
\end{abstract}

Mathematics Subject Classification. 90B30 and 90B50.

Received November 19, 2020. Accepted April 28, 2021.

\section{INTRODUCTION}

With the development of globalization and the increasing variety of commodities, enterprises are facing more and more fierce competition. The introduction of new products is an important means for enterprises to influence the long-term profitability $[9,14,30]$. On the other hand, the increase of environmental pressure also forces manufacturing enterprises to continuously develop environment-friendly new products $[12,34,38,39]$. For

Keywords. Green product diffusion, strategic retailer, game-theoretic analysis, supply chain management, backward induction.

1 School of Management, Hefei University of Technology, Hefei 230009, P.R. China.

2 Key Laboratory of Process Optimization and Intelligent Decision-making of Ministry of Education, Hefei 230009, P.R. China.

3 School of Public Policy and Administration, Chongqing University, Chongqing 400044, P.R. China.

* Corresponding author: gd198410@163.com 
example, Land Rover launched Range Rover new energy vehicle version ${ }^{1}$. BMW currently plans to launch the pure electric version of the 5 series and the pure electric version of the X1, in response to the rapid development of Mercedes Benz and Audi electric R\&D process ${ }^{2}$. Compared with the older generation products, the newer green products often rely on better green attributes, such as lower carbon emissions to attract consumers. However, the success of new products to the market depends not only on the competitiveness of the product itself, but also on its distribution terminals.

Retailers play important roles in the distribution channels $[17,19,26,40]$. With the intensified competition, the emergence of some powerful retailers has had an important impact on manufacturers' product operations. Giant retailers, such as WalMart, might shape the product strategies of the manufacturers that generate the products they need. Such retailers can choose their product marketing independently. At the same time, some retailers with lower scale or market position, while observing the consumer acceptance and profitability of new products, they would also observe the market behavior and product strategies of strong retailers (whether new products are introduced). In other words, retailers with different market power might make differentiated product decisions on whether or not to introduce newer green products due to their strategic behaviors. These behaviors play key roles in affecting the market performance of the newer green generation products in addition to the products' inherent competitiveness. In this context, this paper aims to investigate the following questions:

(1) When the leading retailer introduces green/new generation product, how does the follow-up retailer formulate his product strategies? Should the follow-up retailer choose a same product for Cournot quantity competition or choose a different product for price competition?

(2) As a Stackelberg leader in the supply chain, which product should the leading retailer choose and what pricing/quantity strategy should be developed? Is there such a Pareto area that introducing the green product will benefit both retailers?

In this article, we establish a two-tier supply chain system consisting of a single manufacturer and two competing retailers. The manufacturer sells the older and newer green products through the two retailers. The two retailers are asymmetric in decision sequence, and $\mathrm{R} 1$ takes the lead. The two retailers play dynamically to make strategic product decisions.

This study contributes to the literature by investigating how asymmetric retailers' strategic decision making impacts green supply chain management. For Question (1), our results indicate that in specific intervals, the follow-up retailer might obtain more profit by choosing a different product strategy from the leading retailer to compete in prices; while in other intervals, although imitating the leader's product strategy could only obtain half of the profit of the leader in a quantity competition, it is still more profitable to compete in prices. For Question (2), we derive the Pareto intervals in which introducing a green product could increase profits for both retailers. Results also show that in certain intervals defined by specific thresholds, the introduction of a green product brings profit enhancement to follow-up retailer but loses profit to the leader retailer. In other words, the response behaviors of the follower might force the leading retailer to choose the older generation products. Synthesizing these conclusions, we demonstrate the optimal product selection and pricing strategies of the asymmetric retailers in different scenarios.

The remainder of this paper is organized as follows. Section 2 presents the literature related to our work. Section 3 provides details of the modeling framework. Section 4 demonstrates the equilibrium results and the follow-up retailer's best product strategies are derived. Section 5 shows the possible Pareto improvement of introducing green products on both the retailers. In Section 6, we solve the leader retailer's strategic product selection by considering the follow-up retailer's corresponding responses. Finally, concluding remarks are summarized in Section 7.

\footnotetext{
${ }^{1}$ Available at: https://www.landrover.ca/en/vehicles/phev/index.html.

${ }^{2}$ Available at: http://www.finecars.cc/en/editorial/new-models-article/news/the-2020-bmw-x1-xdrive25e-to-belaunched-followed-by-the-bmw-x2-xdrive25e/index.html?no_cache=1\&tx_ttnews [backPid] =275\&cHash=0c8919d525.
} 


\section{Literature REVIEW}

In this section, we focus on three streams of related literature: (1) Benefits and challenges in new product introduction (NPI). (2) Marketing issues of competing retailers. (3) Supply chain issues on green product, and a summary of literature review is given in Section 2.4.

\subsection{Benefits and challenges in NPI}

Bayus et al. [5] found that firm profitability could benefit from NPI because it can reduce general administrative expenditure. Sorescu et al. [32] investigated the influence of NPI on profits and the risk of the innovating firm, and found that NPI could help to increase firm profit and prevent product lines from being obsolete. Similarly, Palmer and Truong [25] pointed out that there is a positive impact of technological green NPIs on firm profitability. Mehra et al. [22] pointed out that it is common to introduce the upgraded products in a competitive environment, especially for software industry, and they also studied the impact of behavior-based price discrimination on the switching costs and the profits of the incumbent and the entrant. Lobel et al. [20] believed that the expense of a fixed launch cost should be considered whenever the firm decides whether or not to develop new products with new technology level. Nuscheler et al. [23] found that only when the top management teams has more flexible management skills can NPI help technology-based new ventures grow.

NPI is also full of risks. Bianchi [6] believed that the success of a product depends on its market potential rather than whether the design of the product can meet the needs of targeted customers. Moreover, Sok et al. [31] pointed out that the high failure rate for new products lead to high risk of NPI. Baum et al. [4] found that community members activity plays a positive role on the success of NPI. Wang et al. [33] investigated the impact of international diversification on NPI announcements, and found that the value of NPI increases first and then decreases with the increase of international diversification. Khan and Wuest [18] indicated that an upgradable product would change the general value proposition structure of standard products, and manufacturers need to rethink the business model accordingly.

\subsection{Marketing issues of competing retailers}

The price competition among retailers have been extensively studied by existing literature. Brynjolfsson et al. [7] pointed out that one of the key problems of internet business is the competition with traditional physical retailers. They showed that online retailers would facing intense competition from physical retailers when they sell mainstream products. By examining the impacts of channel leadership structures on the strategic interactions between the retailers, Choi and Fred [8] found that the manufacturer should distinguish his national brand product from the retailers' store brands, and deal with the weaker retailer as much as possible. Yang et al. [35] investigated the competition between retailers who compete for both profits and revenue targets. The supply chain system they studied consists of two competing retailers and a common supplier. The results show that when the intensity of price competition increases, the optimal retail price decreases and the optimal order quantity increases. For the Cournot competition among retailers, Fang and Shou [15] study the competition between two supply chains by considering uncertain supply. Results showed that if the opponent retailer's supply becomes unreliable, or if its own supply becomes more reliable, the retailer should order more.

In addition to price competition, supply chain investment is also the focus of retailer competition [2]. Aust and Buscher [3] showed that retailer competition harms all the supply chain members, but is beneficial to the consumers. Similarly, they also found that supply chain leadership is not always advantageous for the manufacturer. It is crucial to participate in the supply chain investment at the right time. Perdikaki et al. [28] investigated the feasible timing of service investments for competing retailers who compete on prices and service levels. Results showed that it is beneficial for retailers to invest in services before the demand is realized. On the retailers' product selection strategies, Pan [26] studied the case when a single retailer is challenged by its manufacturer who is intending to open a direct channel. They showed that one variety would be strategically abandoned by the retailer under certain conditions. 


\subsection{Supply chain issues on green product}

More and more enterprises take the initiative to develop green products because of the enhancement of environmental regulations and consumers' environmental awareness. Palmer and Truong [25] used data on 1020 technological green product introductions to survey whether the firms' profitabilities are positively increased by these innovations. Their results indicated that the relationship between firm profitability and technological green product introduction is positive. Dong et al. [11] pointed out that investing in green product development might not be optimal in the short term, however, it dominates in the long term for supply chain members. In this context, Parsaeifar et al. [27] studied the pricing, recycling, and green investment decisions in a three-echelon supply chain. Both Stackelberg and Nash games are considered when the manufacturer acted as a leader. Under different supply chain structures, Zhu and He [40] found that retailer price competition shows a positive impact on equilibrium greenness, while product greenness competition will reduce equilibrium greenness. The combined impact of price and greenness competition on equilibrium greenness depends on the relative strength of the two competitions.

For the work which are most close to our topic, Agi and Yan [1] studied a supply chain consisting of one retailer and one manufacturer. They demonstrated the conditions under which expanding the conventional product line with a green product is more profitable, and found that it is better for a manufacturer-led supply chain as compared with a retailer-led supply chain to overcome the fixed cost of introducing green products by considering green consumer segment. On green product introduction, Zhang et al. [39] found that high consumer environmental awareness will prompt manufacturers to produce green products, but high consumer environmental awareness does not guarantee the manufacturer to obtain a higher profit. They also showed that the manufacturer's decision on distributing channels has a crucial role in introducing the green products. The key difference of our work as compared to the above two literature is that we try to investigate how does the retailers' strategic and dynamic decisions affect the introduction of a greener new generation product.

\subsection{Summary of the literature review}

The profits generated by producing new products for manufacturers have been widely recognized in both literature and practice. Most of literature on green product diffusion focus on studying the quality performance of newer green products and the recognition degree of consumers. In specific, there is less literature highlight the importance of the market-oriented process on green product introduction, i.e., the role of product distributers like retailers is ignored. To this end, we build a supply chain model with competing retailers to investigate the importance of sellers in green product diffusion. In particular, we observe that retailers do not necessarily participate in the introduction of green products, which can be explained by the asymmetry of retailers and their strategic behaviors. These findings are also in accordance with the reality.

Our research fills the up-mentioned gaps in this area and enriches the importance of retailer decision-making in green product diffusion. The differences between representative related papers and our study are summarized in Table 1 as below.

\section{THE MODELS}

\subsection{Model description}

In this study, we consider a supply chain that consists of one manufacturer and two competing retailers (respectively denoted as R1 and R2). The manufacturer is available to provide two successive generation products, i.e., the older generation product (product a) and the newer green generation product (product b). It is assumed that the new generation product is superior in its green quality as compared to the older generation product a. The two retailers distribute the products and make decisions sequentially. Retailers R1 and R2 are the leaders in the supply chain since retailers play important role in NPI $[8,10,21]$. To model the competition among the two retailers, we assume that retailer $\mathrm{R} 1$ act as the stackelberg leader and retailer $\mathrm{R} 2$ is the game 
TABLE 1. Summary of related papers.

\begin{tabular}{llll}
\hline \hline Research paper & Green product & Retailer competition & Retailer leadership \\
\hline Choi and Fred [8] & $\times$ & $\sqrt{ }$ & $\sqrt{ }$ \\
Yang et al. [35] & $\times$ & $\sqrt{ }$ & $\times$ \\
Aust and Buscher [3] & $\times$ & $\sqrt{ }$ & $\times$ \\
Perdikaki et al. $[28]$ & $\times$ & $\sqrt{ }$ & $\times$ \\
Zhu and He [40] & $\sqrt{ }$ & $\sqrt{ }$ & $\times$ \\
Pan [26] & $\times$ & $\times$ & $\sqrt{ }$ \\
Zhang et al. $[39]$ & $\sqrt{ }$ & $\times$ & $\times$ \\
Agi and Yan [1] & $\sqrt{ }$ & $\times$ & $\times$ \\
Pal and Sarkar [24] & $\sqrt{ }$ & $\times$ & $\times$ \\
This paper & $\sqrt{ }$ & $\sqrt{ }$ & $\sqrt{ }$ \\
\hline
\end{tabular}

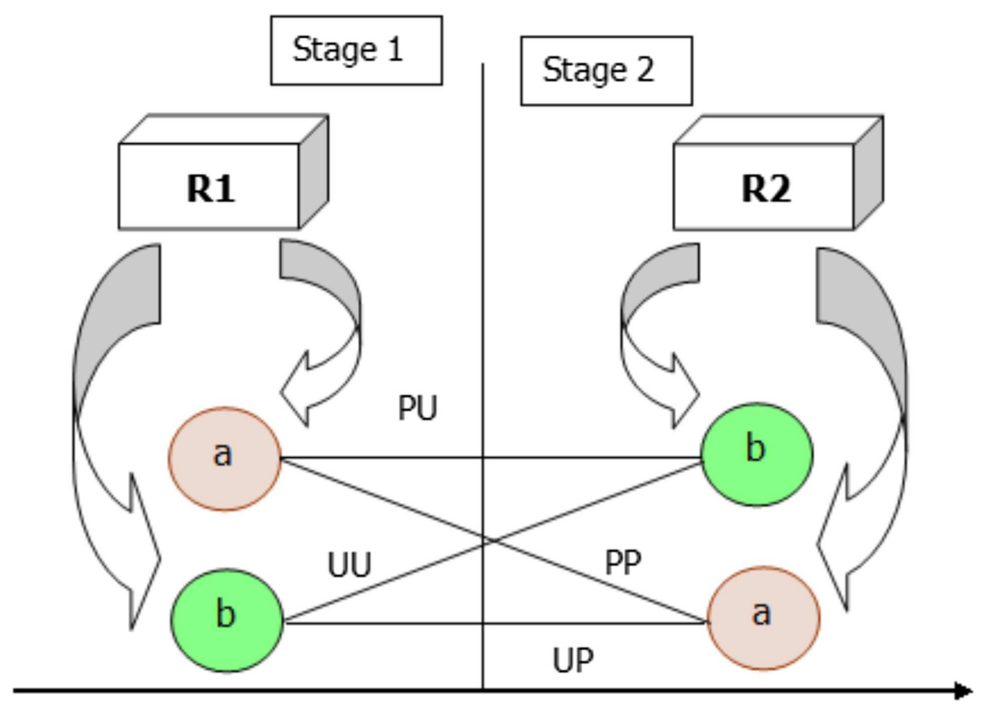

FiguRE 1. Retailers R1 and R2's sequential decisions.

follower. See Figure 1. In the first stage, R1 makes her product strategy and R2 will observe R1's decision and make his own strategic response to maximize his profit $\Pi_{\mathrm{R} 2}$ in the second stage.

In Figure 1, it is clear to show that the strategic choice of retailers would decide whether the green new product could be successfully introduced into the market. For instance, if R1 chooses to sell product b, R1 has to make her product strategy of whether to compete with R1 in prices (chooses to sell product a) or in quantities (chooses to sell product b). The decision problems could be formulated as (1) Retailer R1 decides which product to distribute ( $\mathrm{a}$ or b) and the correspond retail margin $\left(r_{a}\right.$ or $\left.r_{b}\right)$. (2) Retailer R2 observes R1's decisions and make responses on product choice and retail margin $^{3}$. (3) The manufacturer decides the optimal wholesale price(s) $\left(m_{a}\right.$ or $\left.m_{b}\right)$. Thus, there exist four scenarios to consider in the model. With backward induction, the solving technique is: (1) Define the threshold intervals that capture retailer R2' product decisions when R1's product strategy is given (in Sect. 4). (2) Find the overlapping decision range of R2 by comparing the threshold values, and the final product diffusion equilibrium of R1 (and R2) can be found by solving the difference of R1's profits under different product decisions. In the non-overlapping region, we can directly obtain the product

\footnotetext{
${ }^{3}$ It is noted that if $\mathrm{R} 1$ and $\mathrm{R} 2$ choose a same product, the decision problem is to make the optimal quantities $\left(Q_{\mathrm{R} 1}\right.$ and $\left.Q_{\mathrm{R} 2}\right)$.
} 
TABLE 2. Product strategies of the competing retailers.

\begin{tabular}{lll}
\hline \hline Scenarios & $\mathrm{R} 1$ & $\mathrm{R} 2$ \\
\hline $\mathrm{PP}$ & Older generation product & Older generation product \\
UP & New generation product & Older generation product \\
UU & New generation product & New generation product \\
UP & Older generation product & New generation product \\
\hline
\end{tabular}

TABLE 3. Notations.

\begin{tabular}{ll}
\hline \hline Parameters & \\
$U_{i}$ & Consumer net utility of purchasing product $i, i=a, b$. \\
$\theta$ & Consumer preference of older generation product a. \\
$\eta$ & The green increment of product b as compared with product a. \\
$c_{m i}$ & The manufacturer's unit production cost of product $i, i=a, b$. \\
$\Pi_{M}$ & The profit function of the manufacturer. \\
$\Pi_{R j}$ & The profit function of retailer $j, j=1,2$. \\
Decision variables & \\
$r_{i}$ & The retail margin of product $i(i=a, b)$ decided by the retailers. \\
$m_{i}$ & The wholesale price of product $i(i=a, b)$ decided by the manufacturer. \\
$p_{i}$ & The retail price of product $i$. Here, $p_{i}=m_{i}+r_{i}, i=a, b$. \\
\hline
\end{tabular}

equilibrium strategy (in Sect. 6). In Table 2, we show the possible scenarios of this dual-channel supply chain. Next, we will derive the demand functions in different competition scenarios.

\subsection{Demand structure}

It is noted that retailer's demands are decided and interacted by each other's product decisions. In scenario $\mathrm{PP}$, the utility of purchasing an older generation product is $U_{a}^{\mathrm{PP}}=\theta-p_{a}$, and the retailers compete in quantities with the demand function as $Q_{a}^{\mathrm{PP}}=1-p_{a}\left(Q_{a}^{\mathrm{PP}}=Q_{\mathrm{R} 1}^{\mathrm{PP}}+Q_{\mathrm{R} 2}^{\mathrm{PP}}\right.$ denotes the total demand of product a). In scenario UU, the utility of purchasing a upgraded green product is $U_{b}^{\mathrm{UU}}=(1+\eta) \theta-p_{b}$ and we could similarly get the demand as $Q_{b}^{\mathrm{UU}}=1-\frac{p_{b}}{1+\eta}\left(Q_{b}^{\mathrm{UU}}=Q_{\mathrm{R} 1}^{\mathrm{UU}}+Q_{\mathrm{R} 2}^{\mathrm{UU}}\right.$ denotes the total demand of product $\left.\mathrm{b}\right)$. Here, parameter $\theta$ denotes the consumers' preference of product a $(\theta \sim U[0,1]$, see Zhang et al. [39]), and the coefficient $\eta$ denotes the green increment of product $\mathrm{b}$ as compared with product $\mathrm{a}$. It is assumed that the green quality improvement coefficient is exogenously given (see Yenipazarli and Vakharia [36] for a similar setting on modelling the cannibalization between brown and green products).

In scenario PU, the utility of purchasing a product $\mathrm{b}$ is $U_{b}^{\mathrm{PU}}=(1+\eta) \theta-p_{b}$, and the utility of buying a product a is $U_{a}^{\mathrm{PU}}=\theta-p_{a}$. The consumers' indifferent decision point of buying from the two products is $\theta=\frac{p_{b}-p_{a}}{\eta}$. Then, we derive the demands in the presence of prices as $Q_{\mathrm{R} 1}^{\mathrm{PU}}=\frac{p_{b}-p_{a}}{\eta}-p_{a}$ and $Q_{\mathrm{R} 2}^{\mathrm{PU}}=1-\frac{p_{b}-p_{a}}{\eta}$. In this scenario, $\mathrm{R} 1$ and $\mathrm{R} 2$ compete in prices and the reverse price functions are given as $p_{a}=1-Q_{\mathrm{R} 1}^{\mathrm{PU}}-Q_{\mathrm{R} 2}^{\mathrm{PU}}$ and $p_{b}=(\eta+1)\left(1-Q_{\mathrm{R} 1}^{\mathrm{PU}}\right)-Q_{\mathrm{R} 2}^{\mathrm{PU}}$. In scenario UP, we have symmetric price functions: $p_{a}=1-Q_{\mathrm{R} 1}^{\mathrm{UP}}-Q_{\mathrm{R} 2}^{\mathrm{UP}}$ and $p_{b}=(\eta+1)\left(1-Q_{\mathrm{R} 2}^{\mathrm{UP}}\right)-Q_{\mathrm{R} 1}^{\mathrm{UP}}$. The description of notations are summarized in Table 3.

Before describing the analytical model, we discuss the key assumptions specific to capacity and consumer behavior across the two products:

(1) To facilitate our analysis, we assume that the manufacturer has enough production capacity for each of the products. We do not consider the case when there exists a capacity constraint. Similar assumptions have been made in studying new product introduction [37]. 
(2) Shelf constraints have troubled many retail firms $[13,29]$ and retailers are often required to order in a batch ordering form [16]. To tractable survey the retailers' strategic product choices, we assume that each retailer has one unit shelf space to sell one kind of products. That is, a retailer can not sell both products a and b at the same time.

\section{Equilibriums: Strategic Responses of the Follow-UP REtailer}

We solve the supply chain players' optimal decisions with a backward induction framework. In this section, we survey retailer R2's strategic product choice when the pricing or quantity decisions of retailer R1 (the dominant player) is given. As illustrated in Section 3, R1 has two product choices, that is, the green new product b or the old generation product a. In this context, retailer R2, as the follower of R1, also has two choices in stage 2 , that is, to choose the green new product $\mathrm{b}$ or the old generation product a. Without no doubt, this depends on the decision of $\mathrm{R} 1$ in stage 1 . In the next two subsections, we will solve and analyze the optimal decisions of supply chain members (such as R2) when R1 chooses products b or a, respectively.

\subsection{R1 chooses the older generation product}

\subsubsection{Scenario PP: R2 adopts a follow-up product strategy}

In the case when $\mathrm{R} 1$ chooses to sell product a, retailer $\mathrm{R} 2$ has two possible choices. We first survey the scenario when R2 chooses to follow the dominant retailer R1 to sell product a. In this situation, R1 and R2 compete in quantities $\left(Q_{\mathrm{R} 1}\right.$ and $\left.Q_{\mathrm{R} 2}\right)$. The reverse demand function is $p_{a}=1-\left(Q_{\mathrm{R} 1}+Q_{\mathrm{R} 2}\right)$, with $p_{a}=m_{a}+r_{a}$. The profit function of the manufacturer is given as:

$$
\max \Pi_{M}^{\mathrm{PP}}\left(m_{a} \mid Q_{\mathrm{R} 1}, Q_{\mathrm{R} 2}\right)=\left(Q_{\mathrm{R} 1}+Q_{\mathrm{R} 2}\right)\left(m_{a}-c_{m a}\right)=\left(-m_{a}-r_{a}+1\right)\left(m_{a}-c_{m a}\right) .
$$

The profit functions of the two retailers are given as follows:

$$
\begin{aligned}
\max \Pi_{\mathrm{R} 2}^{\mathrm{PP}}\left(Q_{\mathrm{R} 2} \mid Q_{\mathrm{R} 1}\right) & =Q_{\mathrm{R} 2}\left(-m_{a}+p_{a}\right) \\
\max \Pi_{\mathrm{R} 1}^{\mathrm{PP}}\left(Q_{\mathrm{R} 1}\right) & =Q_{\mathrm{R} 1}\left(-m_{a}+p_{a}\right) .
\end{aligned}
$$

In equation (4.1), the term $\left(m_{a}-c_{m a}\right)$ denotes the manufacturer's unit net revenue form product a. In equations (4.2) and (4.3), the term $\left(-m_{a}+p_{a}\right)$ is the retailers' unit net income from selling product a.

Lemma 4.1. When $R 1$ chooses product a, retailers $R 1$ and R2 play dynamically in a Stackelberg-cournot quantity game, by using backward induction, the equilibrium results of scenarios PP and PU are given in Table 4. Here, $\tilde{\eta}=\eta+1$.

\subsubsection{Scenario PU: R2 chooses the newer green product}

Next, we further investigate when R2 chooses a different product strategy with retailer R1 who chooses product a, that is to say, retailer R2 sells the new generation product $\mathrm{b}$. In this situation, the two retailers compete in prices. The profit of the manufacturer is expressed as:

$$
\begin{aligned}
\max \Pi_{M}^{\mathrm{PU}}\left(m_{a}, m_{b} \mid r_{a}, r_{b}\right)= & Q_{\mathrm{R} 2}\left(m_{b}-c_{m b}\right)+Q_{\mathrm{R} 1}\left(m_{a}-c_{m a}\right) \\
= & \left(1-\frac{\left(m_{b}+r_{b}\right)-\left(m_{a}+r_{a}\right)}{\eta}\right)\left(m_{b}-c_{m b}\right) \\
& +\left(\frac{\left(m_{b}+r_{b}\right)-\left(m_{a}+r_{a}\right)}{\eta}-\left(m_{a}+r_{a}\right)\right)\left(m_{a}-c_{m a}\right)
\end{aligned}
$$

and the two retailers' profit functions are given as follows: 
TABLE 4. The equilibriums when $\mathrm{R} 1$ chooses product a.

\begin{tabular}{|c|c|c|}
\hline Optimum & $\mathrm{PP}$ & $\mathrm{PU}$ \\
\hline Price & $\begin{array}{l}m_{a}^{\mathrm{PP}^{*}}=\frac{1}{7}\left(4 c_{m a}+3\right) \\
r_{a}^{\mathrm{PP}^{*}}=\frac{1}{7}\left(1-c_{m a}\right)\end{array}$ & $\begin{array}{l}m_{b}^{\mathrm{PU}^{*}}=\frac{1}{4}\left(\frac{(\eta+1)\left(-c_{m a}+2 \eta+2\right)}{2 \eta+1}+3 c_{m b}\right) \\
m_{a}^{\mathrm{PU}^{*}}=\frac{1}{8}\left(\frac{(12 \eta+5) c_{m a}+6 \eta+4}{2 \eta+1}-\frac{c_{m b}}{\eta+1}\right) \\
r_{b}^{\mathrm{PU}^{*}}=\frac{1}{2}\left(\frac{(\eta+1)\left(c_{m a}+2 \eta\right)}{2 \eta+1}-c_{m b}\right) \\
r_{a}^{\mathrm{PU}^{*}}=\frac{1}{4}\left(\frac{2 \eta-(4 \eta+1) c_{m a}}{2 \eta+1}+\frac{c_{m b}}{\eta+1}\right)\end{array}$ \\
\hline Demand & $\begin{array}{l}Q_{\mathrm{R} 1}^{\mathrm{PP}^{*}}=\frac{2}{7}\left(1-c_{m a}\right) \\
Q_{\mathrm{R} 2}^{\mathrm{PP}}=\frac{1}{7}\left(1-c_{m a}\right)\end{array}$ & $\begin{aligned} Q_{\mathrm{R} 1}^{\mathrm{PU}^{*}} & =\frac{(\eta+1)\left(c_{m a}+2 \eta\right)-(2 \eta+1) c_{m b}}{8 \eta(\eta+1)} \\
Q_{\mathrm{R} 2}^{\mathrm{PU}^{*}} & =\frac{(2 \eta+1) c_{m b}-(4 \eta+1)(\eta+1) c_{m a}+2 \eta(\eta+1)}{8 \eta(2 \eta+1)}\end{aligned}$ \\
\hline Profit & $\begin{array}{l}\Pi_{\mathrm{R} 1}^{\mathrm{PP}}=\frac{2}{49}\left(1-c_{m a}\right)^{2} \\
\Pi_{\mathrm{R} 2}^{\mathrm{PP}^{*}}=\frac{1}{49}\left(1-c_{m a}\right)^{2} \\
\Pi_{M}^{\mathrm{PP}}=\frac{9}{49}\left(1-c_{m a}\right)^{2}\end{array}$ & $\begin{aligned} \Pi_{\mathrm{R} 1}^{\mathrm{PU}}= & \frac{\left((\eta+1) c_{m a}-(2 \eta+1) c_{m b}+2 \eta(\eta+1)\right)^{2}}{16 \eta(\eta+1)(2 \eta+1)} \\
\Pi_{\mathrm{R} 2}^{\mathrm{PU}}= & \frac{\left((2 \eta+1) c_{m b}-\left(4 \eta^{2}+5 \eta+1\right) c_{m a}+2 \eta(\eta+1)\right)^{2}}{32 \eta(\eta+1)(2 \eta+1)^{2}} \\
\Pi_{M}^{\mathrm{PU}}= & \frac{\left(16 \eta^{2}+12 \eta+1\right) \tilde{\eta}^{2} c_{m a}^{2}-2 \tilde{\eta} c_{m a}(\eta+\tilde{\eta}) c_{m b}-4 \eta \tilde{\eta}\left(8 \eta^{2}+10 \eta+3\right) c_{m b}}{64 \eta \tilde{\eta}(2 \eta+1)^{2}} \\
& \quad+\frac{2 \eta \tilde{\eta}(8 \eta+5)+(2 \eta+1)^{2}(4 \eta+1) c_{m b}^{2}+4 \eta\left(4 \eta^{2}+9 \eta+4\right) \tilde{\eta}^{2}}{64 \eta \tilde{\eta}(2 \eta+1)^{2}}\end{aligned}$ \\
\hline
\end{tabular}

$$
\begin{aligned}
\max \Pi_{\mathrm{R} 2}^{\mathrm{PU}}\left(r_{b} \mid r_{a}\right) & =Q_{\mathrm{R} 2} r_{b}=r_{b}\left(1-\frac{\left(m_{b}+r_{b}\right)-\left(m_{a}+r_{a}\right)}{\eta}\right) \\
\max \Pi_{\mathrm{R} 1}^{\mathrm{PU}}\left(r_{a}\right) & =Q_{\mathrm{R} 1} r_{a}=r_{a}\left(\frac{\left(m_{b}+r_{b}\right)-\left(m_{a}+r_{a}\right)}{\eta}-\left(m_{a}+r_{a}\right)\right) .
\end{aligned}
$$

In this scenario, R2 observes R1's price decision $r_{a}$ and makes his response pricing of green product $\left(r_{b}\right)$. After the retailers' price competition, the manufacturer decides the wholesale prices $m_{a}$ and $m_{b}$. The equilibrium results are summarized in Table 4.

To ensure both the demands of products $\mathrm{a}$ and $\mathrm{b}$ are positive, it is straightforward to require that $c_{m b} \in$ $\left(\frac{(\eta+1)\left((4 \eta+1) c_{m a}-2 \eta\right)}{2 \eta+1}, \frac{(\eta+1)\left(c_{m a}+2 \eta\right)}{2 \eta+1}\right)$. In other words, the unit cost of green product b could not be too large $\left(c_{m b}>\frac{(\eta+1)\left(c_{m a}+2 \eta\right)}{2 \eta+1}\right.$ leads to $\left.Q_{\mathrm{R} 2}^{\mathrm{PU}^{*}}<0\right)$ or too small $\left(c_{m b}<\frac{(\eta+1)\left((4 \eta+1) c_{m a}-2 \eta\right)}{2 \eta+1}\right.$ leads to $\left.Q_{\mathrm{R} 1}^{\mathrm{PU}^{*}}<0\right)$. The setting of upper and lower bound would help to accurately analyze the strategic decisions of the two retailers. After giving the optimal equilibrium results under the two strategies (in Sects. 4.1.1 and 4.1.2), the follow-up retailer (retailer R2) needs to make strategic product selection of his own. Next we analyze how R2 weighs the two strategies to make his ex-post decision when $\mathrm{R} 1$ chooses product a.

\subsubsection{R2's strategic choice: PP or PU?}

The following proposition is proposed to illustrate how retailer R2 make his strategic product choice.

Proposition 4.2. We have two cases to consider:

(1) If $\eta>\frac{2}{49}$, when $\bar{c}_{m b, 1}<c_{m b}<\tilde{c}_{m b, 1}$, retailer $R 2$ would choose to sell the newer green generation product $b$; when $\tilde{c}_{m b, 1}<c_{m b}<\bar{c}_{m b, 2}$, retailer $R 2$ would choose to sell the older generation product $a$.

(2) If $\eta \leq \frac{2}{49}$, retailer $R 2$ should choose to sell the older generation product a all the time. 


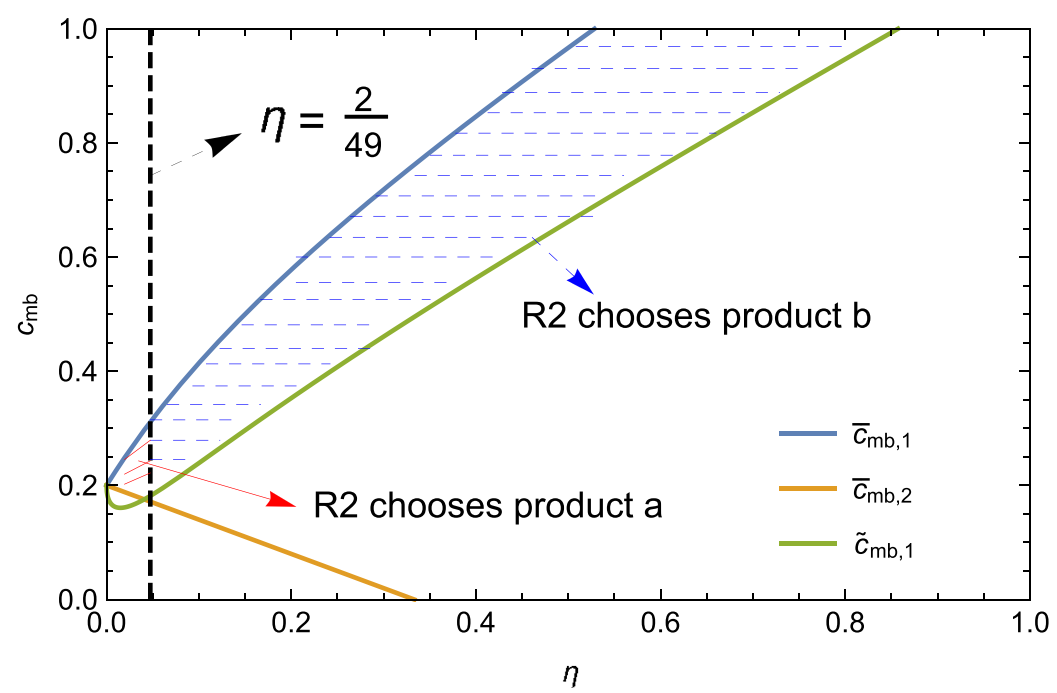

Figure 2. Retailer R2's strategic product choice when R1 chooses product a (with $c_{m a}=0.2$ ).

$$
\text { Here, } \bar{c}_{m b, 1}=(2 \eta+1) c_{m a}-\eta, \bar{c}_{m b, 2}=\frac{(2 \eta+1) c_{m a}+\eta(4 \eta+3)}{4 \eta+1} \text { and } \tilde{c}_{m b, 1}=\frac{7 \eta(4 \eta+3)-(2 \eta+1)\left(4 \sqrt{2 \eta}\left(c_{m a}-1\right)-7 c_{m a}\right)}{7(4 \eta+1)} \text {. }
$$

First, we find that when the consumer's recognition of the new generation product $\mathrm{b}$ is large enough $\left(\eta>\frac{2}{49}\right)$, retailer R2 needs to choose between two product strategies: if the unit cost of green product $\mathrm{b}$ is low $\left(\bar{c}_{m b, 1}<\right.$ $\left.c_{m b}<\tilde{c}_{m b, 1}\right)$, he would choose the green product and retailer R1 for price competition, and when the unit cost of the green product is too high $\left(\tilde{c}_{m b, 1}<c_{m b}<\bar{c}_{m b, 2}\right)$, he would compete with retailer R1 for quantity competition to choose the older generation of product a. Second, when the consumer's recognition of the new generation of products is not high enough $\left(\eta \leq \frac{2}{49}\right)$, retailer R2 has to choose the older generation product a regardless of the cost of product $\mathrm{b}$. This finding implies that the follow-up retailer should follow the leading retailer's product strategy when the added greenness is not competitive for a price competition with the older generation products. A numerical example is shown in Figure 2.

In addition, we find that the difference $\Pi_{\mathrm{R} 2}^{\mathrm{PU}}-\Pi_{\mathrm{R} 2}^{\mathrm{PP}}$ is a convex function of product b's unit cost $c_{m, b}$. There also exists a threshold value $c_{m b}=\frac{(2 \eta+1) c_{m a}+\eta(4 \eta+3)}{4 \eta+1}$, such that when $c_{m, b}<\frac{(2 \eta+1) c_{m a}+\eta(4 \eta+3)}{4 \eta+1}, \Pi_{\mathrm{R} 2}^{\mathrm{PU}}-\Pi_{\mathrm{R} 2}^{\mathrm{PP}}$ is decreasing with $c_{m, b}$, and when $c_{m, b} \geq \frac{(2 \eta+1) c_{m a}+\eta(4 \eta+3)}{4 \eta+1}, \Pi_{\mathrm{R} 2}^{\mathrm{PU}}-\Pi_{\mathrm{R} 2}^{\mathrm{PP}}$ is increasing with $c_{m, b}$. Note that the upper limit of the nonnegative constraint $\bar{c}_{m b, 2}$ is smaller than the axis of symmetry $\frac{(2 \eta+1) c_{m a}+\eta(4 \eta+3)}{4 \eta+1}$, thus the difference function $\Pi_{\mathrm{R} 2}^{\mathrm{PU}}-\Pi_{\mathrm{R} 2}^{\mathrm{PP}}$ is decreasing with $c_{m, b}$ all the time. In the above analysis, we investigate the scenarios that the dominant retailer (R1) conservatively chooses the older generation product a, and provide the optimal strategy of retailer R2 as a follower. In the next section, we will survey the strategic decision of retailer $\mathrm{R} 2$ when the dominant retailer (R1) chooses the newer green product $\mathrm{b}$.

\section{2. $\mathrm{R} 1$ chooses the newer green generation product}

\subsubsection{Scenario UU: R2 adopts a follow-up strategy}

When $\mathrm{R} 1$ chooses to sell product b, retailer R2 has two choices. We first study the scenario when R2 chooses to follow the dominant retailer $\mathrm{R} 1$ to sell the new generation product $\mathrm{b}$. In this case, the two retailers competes in quantities. The reverse demand function is $p_{b}=(\eta+1)\left(1-\left(Q_{\mathrm{R} 1}+Q_{\mathrm{R} 2}\right)\right)$, here $p_{b}=m_{b}+r_{b}$. The profit 
TABLE 5. The equilibriums when $\mathrm{R} 1$ chooses product $\mathrm{b}$.

\begin{tabular}{|c|c|c|}
\hline Results & UU & UP \\
\hline Price & $\begin{array}{l}m_{b}^{\mathrm{UU}^{*}}=\frac{4 c_{m b}+3 \eta+3}{7} \\
r_{b}^{\mathrm{UU}^{*}}=\frac{1+\eta-c_{m b}}{7}\end{array}$ & $\begin{array}{l}m_{a}^{\mathrm{UP}^{*}}=\frac{(6 \eta+3) c_{m a}-c_{m b}+3 \eta+2}{8 \eta+4} \\
m_{b}^{\mathrm{UP}^{*}}=\frac{(12 \eta+5) c_{m b}-(2 \eta+1) c_{m a}+\eta(4 \eta+9)+4}{16 \eta+8} \\
r_{a}^{\mathrm{UP}^{*}}=\frac{-(2 \eta+1) c_{m a}+c_{m b}+\eta}{4 \eta+2} \\
r_{b}^{\mathrm{UP}^{*}}=\frac{(2 \eta+1) c_{m a}-(4 \eta+1) c_{m b}+\eta(4 \eta+3)}{8 \eta+4}\end{array}$ \\
\hline Demand & $\begin{aligned} Q_{\mathrm{R} 1}^{\mathrm{UU}^{*}} & =\frac{2}{7}-\frac{2 c_{m b}}{7(\eta+1)} \\
Q_{\mathrm{R} 2}^{\mathrm{UU}^{*}} & =\frac{1+\eta-c_{m b}}{7 \eta+7}\end{aligned}$ & $\begin{aligned} Q_{\mathrm{R} 1}^{*} & =\frac{-(2 \eta+1) c_{m a}+c_{m b}+\eta}{8 \eta} \\
Q_{\mathrm{R} 2}^{\mathrm{UP}^{*}} & =\frac{2 \eta c_{m a}+c_{m a}-(4 \eta+1) c_{m b}+4 \eta^{2}+3 \eta}{8 \eta(2 \eta+1)}\end{aligned}$ \\
\hline Profits & $\begin{array}{l}\Pi_{\mathrm{R} 1}^{\mathrm{UU}^{*}}=\frac{2\left(1+\eta-c_{m b}\right)^{2}}{49(\eta+1)} \\
\Pi_{\mathrm{R} 2}^{\mathrm{UU}^{*}}=\frac{\left(1+\eta-c_{m b}\right)^{2}}{49(\eta+1)} \\
\Pi_{M}^{\mathrm{UU}^{*}}=\frac{9\left(1+\eta-c_{m b}\right)^{2}}{49(\eta+1)}\end{array}$ & $\begin{aligned} \Pi_{\mathrm{R} 1}^{\mathrm{UP}}= & \frac{\left(\eta-2 \eta c_{m a}-c_{m a}+c_{m b}\right)^{2}}{32 \eta^{2}+16 \eta} \\
\Pi_{\mathrm{R} 2}^{\mathrm{UP}}= & \frac{\left(2 \eta c_{m a}+c_{m a}-4 \eta c_{m b}-c_{m b}+4 \eta^{2}+3 \eta\right)^{2}}{32 \eta(2 \eta+1)^{2}} \\
\Pi_{M}^{\mathrm{UP}}= & \frac{(\eta+\tilde{\eta})^{2}(4 \eta+1) c_{m a}^{2}+(4 \eta(4 \eta+3)+1) c_{m b}^{2}-2 \eta(4 \eta(4 \tilde{\eta}+3)+11) c_{m b}}{64 \eta(2 \eta+1)^{2}} \\
& +\frac{\eta(\eta(4 \eta(4 \eta+15)+57)+16)-2(2 \eta+1)\left(c_{m b}+\eta(8 \eta+5)\right) c_{m a}}{64 \eta(2 \eta+1)^{2}}\end{aligned}$ \\
\hline
\end{tabular}

function of the manufacturer is given as follows:

$$
\max \Pi_{M}^{\mathrm{UU}}\left(m_{b} \mid Q_{\mathrm{R} 1}, Q_{\mathrm{R} 2}\right)=\left(Q_{\mathrm{R} 1}+Q_{\mathrm{R} 2}\right)\left(m_{b}-c_{m b}\right)=\left(1-\frac{m_{b}+r_{b}}{\eta+1}\right)\left(m_{b}-c_{m b}\right)
$$

and retailers R1 and R2's profit functions are given as follows

$$
\begin{aligned}
\max \Pi_{\mathrm{R} 2}^{\mathrm{UU}}\left(Q_{\mathrm{R} 2} \mid Q_{\mathrm{R} 1}\right) & =Q_{\mathrm{R} 2}\left(-m_{b}+p_{b}\right) \\
\max \Pi_{\mathrm{R} 1}^{\mathrm{UU}}\left(Q_{\mathrm{R} 1}\right) & =Q_{\mathrm{R} 1}\left(-m_{b}+p_{b}\right) .
\end{aligned}
$$

In equation (4.7), the term $\left(m_{b}-c_{m b}\right)$ denotes the manufacturer's unit net revenue form producing green product b. In equations (4.8) and (4.9), the term $\left(-m_{b}+p_{b}\right)$ denotes the unit retail income from selling green product $b$.

Lemma 4.3. When $R 1$ chooses product $b$, retailers $R 1$ and R2 play dynamically in a Stackelberg-cournot quantity game, by using backward induction, the equilibrium results of scenarios UU and UP are given in Table 5. Here, $\tilde{\eta}=\eta+1$.

\subsubsection{Scenario UP: R2 chooses the older generation product}

Next, we further investigate when R2 chooses a different product strategy from retailer R1, that is to say, retailer $\mathrm{R} 2$ chooses the older generation product a. In this condition, the two retailers compete in prices to maximize their profits.

We first give the profit function the manufacturer as:

$$
\begin{aligned}
\max \Pi_{M}^{U P}\left(m_{a}, m_{b} \mid r_{a}, r_{b}\right) & =Q_{\mathrm{R} 2}\left(m_{a}-c_{m a}\right)+Q_{\mathrm{R} 1}\left(m_{b}-c_{m b}\right) \\
& =\left(\frac{\left(m_{b}+r_{b}\right)-\left(m_{a}+r_{a}\right)}{\eta}-\left(m_{a}+r_{a}\right)\right)\left(m_{a}-c_{m a}\right)
\end{aligned}
$$




$$
+\left(1-\frac{\left(m_{b}+r_{b}\right)-\left(m_{a}+r_{a}\right)}{\eta}\right)\left(m_{b}-c_{m b}\right)
$$

and the two retailers' profit functions are given as follows:

$$
\begin{aligned}
\max \Pi_{\mathrm{R} 2}^{U P}\left(r_{a} \mid r_{b}\right) & =Q_{\mathrm{R} 2} r_{a}=\left(\frac{\left(m_{b}+r_{b}\right)-\left(m_{a}+r_{a}\right)}{\eta}-\left(m_{a}+r_{a}\right)\right) r_{b} \\
\max \Pi_{\mathrm{R} 1}^{U P}\left(r_{b}\right) & =Q_{\mathrm{R} 1} r_{b}=\left(1-\frac{\left(m_{b}+r_{b}\right)-\left(m_{a}+r_{a}\right)}{\eta}\right) r_{a} .
\end{aligned}
$$

By using backward induction, the equilibrium results are also summarized in Table 5.

To ensure both the demands of products $\mathrm{a}$ and $\mathrm{b}$ are positive, it is direct to require that $c_{m b} \in$ $\left(c_{m a}(2 \eta+1)-\eta, \frac{(2 \eta+1) c_{m a}+\eta(4 \eta+3)}{4 \eta+1}\right)$. In other words, the unit cost of product $\mathrm{b}$ cannot be too large or too small. The setting of upper and lower bound would help to accurately analyze the strategic decisions of the two retailers. After giving the optimal equilibrium results under the two strategies (given in Sects. 4.2 .1 and 4.2.2), the follow-up retailer R2 needs to make his own strategic product selection.

\subsubsection{R2's strategic choice: UU or UP?}

In this section, we analyze how R2 weighs the two strategies to make his ex-post decision when R1 chooses product $b$.

Proposition 4.4. We have four cases to consider:

(1) If $0<\eta<\frac{2}{49}$, R2 would choose to sell the new generation product when $\bar{c}_{m b, 3}<c_{m b}<\tilde{c}_{m b, 3}$ and choose to sell product a when $\tilde{c}_{m b, 3}<c_{m b}<\bar{c}_{m b, 4}$.

(2) If $\frac{2}{49} \leq \eta<\frac{49}{32}$, R2 would choose to sell the newer green generation product all the time.

(3) If $\eta>\frac{49}{32}$, R2 should choose to sell the new generation product when $\bar{c}_{m b, 3}<c_{m b}<\tilde{c}_{m b, 3}$, and R2 should choose to sell the older generation product when $\tilde{c}_{m b, 3}<c_{m b}<\bar{c}_{m b, 4}$.

(4) If $\eta=\frac{49}{32}$, R2 should choose to sell the newer green generation product when $\bar{c}_{m b, 3}<c_{m b}<\frac{81\left(57 c_{m a}+8\right)}{2080}$; and R2 would choose to sell the older generation product when $\frac{81\left(57 c_{m a}+8\right)}{2080}<c_{m b}<\bar{c}_{m b, 4}$ is satisfied.

$$
\begin{gathered}
\text { Here, }, \bar{c}_{m b, 3} \\
\frac{(\eta+1)\left(c_{m a}+2 \eta\right)}{2 \eta+1} .
\end{gathered}=\frac{(\eta+1)\left((4 \eta+1) c_{m a}-2 \eta\right)}{2 \eta+1}, \tilde{c}_{m b, 3}=\frac{(\eta+1)\left(2 \eta(32 \eta+65)-7(4 \eta+1)\left(7 c_{m a}-\frac{4 \sqrt{2}\left(c_{m a}-1\right)}{\sqrt{\frac{1}{\eta}}}\right)\right)}{(2 \eta+1)(32 \eta-49)} \text { and } \bar{c}_{m b, 4}=
$$

In Proposition 4.4, we present the follow-up retailer R2's strategic product choices when the dominant retailer $\mathrm{R} 1$ chooses to sell the green product b. It can be found that there exist thresholds of consumer preference $\eta$ $\left(\eta=\frac{2}{49}\right.$ and $\left.\eta=\frac{49}{32}\right)$ and production cost $c_{m b}\left(\bar{c}_{m b, 3}, \bar{c}_{m b, 4}\right.$ and $\left.\tilde{c}_{m b, 3}\right)$ that define R2's strategic choice. Specially, when the consumer preference for the greener product locates in the interval $\left(\frac{2}{49} \leq \eta<\frac{49}{32}\right)$, retailer R2 would always obtain a larger profit in scenario $\mathrm{UU}$ as compared to the profit in scenario $\mathrm{UP}\left(\Pi_{\mathrm{R} 2}^{\mathrm{UU}}{ }^{*}>\Pi_{\mathrm{R} 2}^{\mathrm{UP}^{*}}\right)$, and he will choose the new generation product all the time. This finding is not in accordance with the finding in Proposition 4.2. This indicates that when retailer $\mathrm{R} 1$ chooses to sell product $\mathrm{b}$, there does not exist a interval of $\eta$, in which $\mathrm{R} 2$ will always choose product a when product cost $c_{m b}$ varies.

When $0<\eta<\frac{2}{49}$, R2 would choose to sell product b only if $c_{m b}$ is lower than the threshold $\tilde{c}_{m b, 3}$, and choose to sell the older generation product when $c_{m b}$ is larger than $\tilde{c}_{m b, 3}\left(\tilde{c}_{m b, 3}\right.$ is the larger of the two roots of $\Pi_{\mathrm{R} 2}^{\mathrm{UU}^{*}}-\Pi_{\mathrm{R} 2}^{\mathrm{UP}}=0$ when $\left.0<\eta<\frac{49}{32}\right)$. This finding is also not in line with Proposition 4.2 since the function $\Pi_{\mathrm{R} 2}^{\mathrm{UU}^{*}}-\Pi_{\mathrm{R} 2}^{\mathrm{UP}}$ is concave with $c_{m b}$ when $0<\eta<\frac{2}{49}$. However, when consumer preference $\eta$ is larger enough $\left(\eta>\frac{49}{32}\right), \mathrm{R} 2$ will choose to sell green product $\mathrm{b}$ only if $c_{m b}$ is lower than the threshold $\tilde{c}_{m b, 3}\left(\tilde{c}_{m b, 3}\right.$ is the smaller of the two roots of $\Pi_{\mathrm{R} 2}^{\mathrm{UU}^{*}}-\Pi_{\mathrm{R} 2}^{\mathrm{UP}}=0$ when $\left.\eta>\frac{49}{32}\right)$, and choose to sell the older generation product when $c_{m b}$ is larger than $\tilde{c}_{m b, 3}$. This finding is in line with Proposition 4.2. This phenomenon could be explained that 


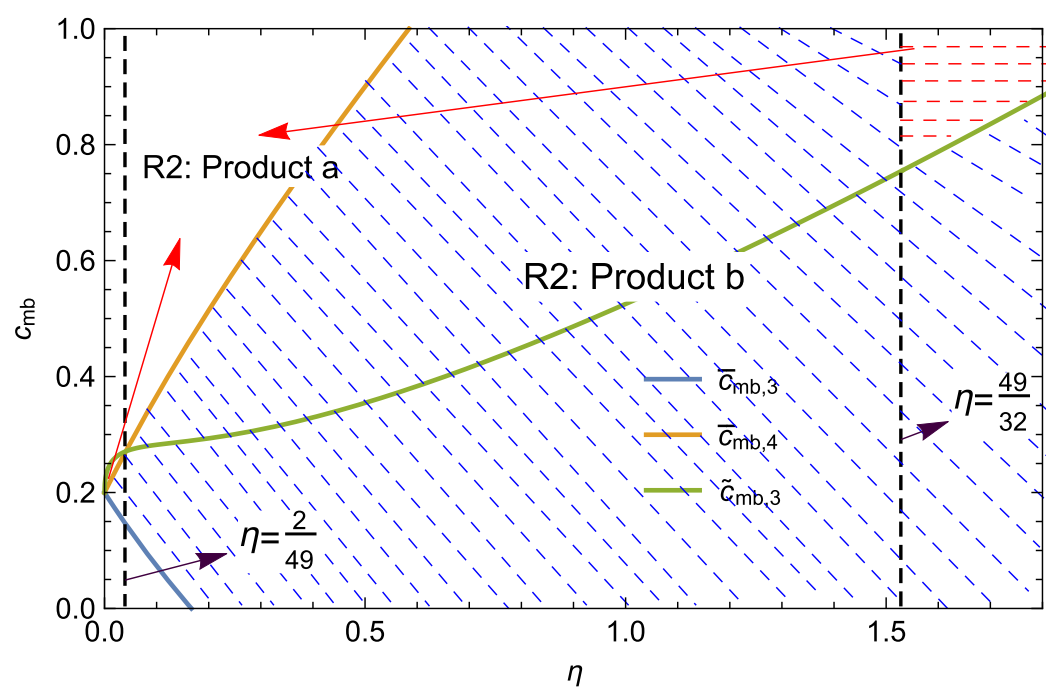

FiguRE 3. Retailer R2's strategic product choice when R1 chooses product $\mathrm{b}$ (with $c_{m a}=0.2$ and $\left.\eta \neq \frac{49}{32}\right)$.

even if consumers have a high recognition of product $\mathrm{b}$, retailer $\mathrm{R} 2$ will not choose to sell newer green products if the marginal income of selling product $\mathrm{b}$ is not high enough (because of a high $c_{m b}$ ). This finding suggests that for the retailer with a low market position (e.g., a follower), even if consumers have a high degree of green recognition, when the upstream manufacturer's production efficiency is not high enough, choosing traditional products is still the better choice.

We also show that there exists a special case that when $\eta=\frac{49}{32}$, the profit difference of $\Pi_{\mathrm{R} 2}^{\mathrm{UU}}-\Pi_{\mathrm{R} 2}^{\mathrm{UP}^{*}}$ is linear decreasing function of $c_{m b}$ and $\Pi_{\mathrm{R} 2}^{\mathrm{UU}^{*}}-\Pi_{\mathrm{R} 2}^{\mathrm{UP}}=0$ if $c_{m b}=\frac{81\left(57 c_{m a}+8\right)}{2080}$. When $\eta \neq \frac{49}{32}$, by solving the axis of $\Pi_{\mathrm{R} 2}^{\mathrm{UU}^{*}}-\Pi_{\mathrm{R} 2}^{\mathrm{UP}}$, we have $c_{m b}=\frac{(\eta+1)\left(2 \eta(32 \eta+65)-49(4 \eta+1) c_{m a}\right)}{(2 \eta+1)(32 \eta-49)}$. Further more, we find that the upper bound $\bar{c}_{m b, 4}$ is smaller than the axis $\left(\frac{(\eta+1)\left(c_{m a}+2 \eta\right)}{2 \eta+1}<\frac{(\eta+1)\left(2 \eta(32 \eta+65)-49(4 \eta+1) c_{m a}\right)}{(2 \eta+1)(32 \eta-49)}\right)$ in the case when $\eta>\frac{49}{32}$, this indicates that the profit difference between retailer R2's product choices $\left(\Pi_{\mathrm{R} 2}^{\mathrm{UU}^{*}}-\Pi_{\mathrm{R} 2}^{\mathrm{UP}}\right)$ is always decreasing with $c_{m b}$ when $\eta>\frac{49}{32}$, and always increasing with $c_{m b}$ when $\eta<\frac{49}{32}$. In a word, when $\eta \geq \frac{49}{32}$, the difference $\Pi_{\mathrm{R} 2}^{\mathrm{UU}^{*}}-\Pi_{\mathrm{R} 2}^{\mathrm{UP}^{*}}$ is always decreasing with $c_{m b}$.

In Figures 3 and 4 , we respectively show the case when $\eta \neq \frac{49}{32}$ and $\eta=\frac{49}{32}$. By observing Figure 3, we find that as compared to the case when R1 chooses product a (shown in Fig. 2), the zone in which R2 chooses green product $\mathrm{b}$ is largely increased when $\mathrm{R} 1$ chooses to sell product $\mathrm{b}$ (the blue area). In Figure 4, we illustrate R2's strategic choice when $c_{m a}$ as $\eta$ is deterministic $\left(\eta=\frac{49}{32}\right)$. Results show that when $c_{m b}$ is too large (the red area), $\mathrm{R} 2$ should choose to sell product a. Only when $c_{m b}$ is not too large (the blue area), R2 should choose to sell the new product $\mathrm{b}^{4}$.

\section{Pareto improvement of NeW Product introduction}

In this section, we will survey the Pareto improvements on both retailers R1 and R2's profit. We start from the individual action of retailer R2 to analyze the profit increase brought by the introduction of green product, that is, only retailer R2 adopts to sell the new generation product b. The competitiveness of adopting product

\footnotetext{
${ }^{4}$ The threshold $\bar{c}_{m b, 4}$ is always larger than 1 when $\eta=\frac{49}{32}$, and thus the line of $\bar{c}_{m b, 4}$ does not appear in Figure 3.
} 


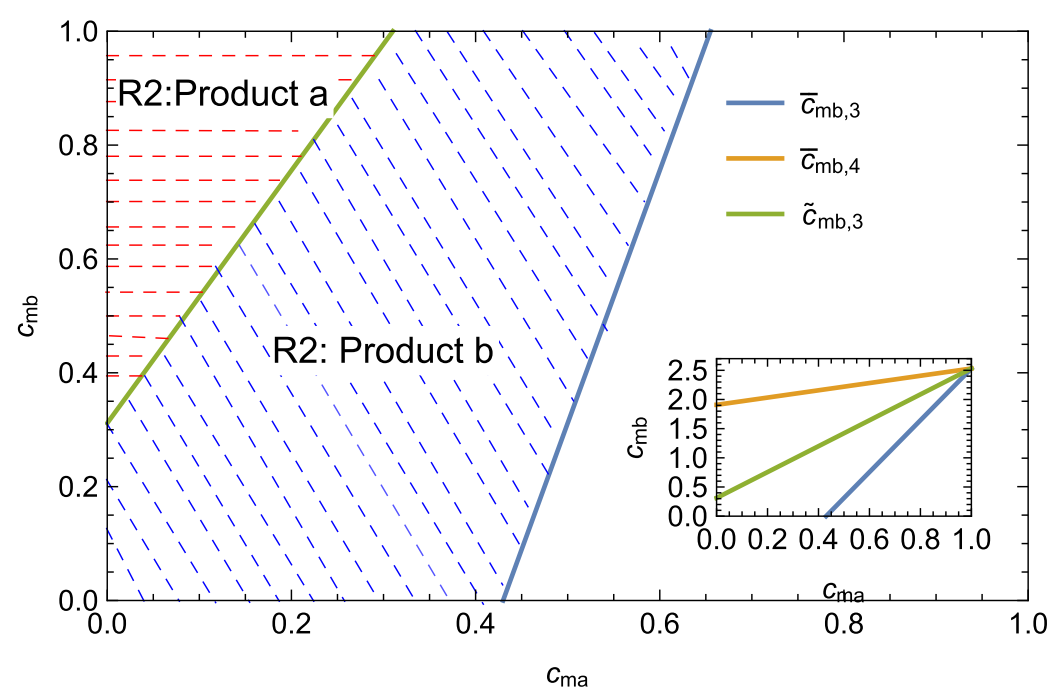

FIGURE 4. Retailer R2's strategic product choice when R1 chooses product $\mathrm{b}$ (with $\eta=\frac{49}{32}$ ).

b on R2's profit has been given in Proposition 4.2, and then, by comparing profits $\Pi_{\mathrm{R} 1}^{\mathrm{PU}^{*}}$ and $\Pi_{\mathrm{R} 1}^{\mathrm{PP}^{*}}$, we have the following findings:

Proposition 5.1. In scenario PU, retailer $R 1$ always obtain a smaller profit when $R 2$ chooses product b, i.e., $R 1$ and R2 could not reach a Pareto in profit at the same time.

In scenario PU, retailer R1 is inactive to choose the green product while the follow-up retailer R2 is active to choose the green product. As compared to scenario PP, in which both the two retailers sell product a, we show the "extra" profit brought by retailer R2's active product strategy in Proposition 5.1. Results indicate that the two retailers could not reach a Pareto improvement on profits in scenario PU. As shown in Proposition 4.2, the conditions $\eta>\frac{2}{49}$ and $\bar{c}_{m b, 1}<c_{m b}<\tilde{c}_{m b, 1}$ ensure retailer R2 to choose product b with a larger profit as compared to his profit in the benchmark scenario $\mathrm{PP}\left(\Pi_{\mathrm{R} 2}^{\mathrm{PU}^{*}}-\Pi_{\mathrm{R} 2}^{\mathrm{PP}}>0\right)$. On comparing retailer R1's profits in scenarios PU and PP, we find that retailer R1 always obtain a smaller profit in PU as compared with the profit in scenario PP. Figure 5 depicts the performance of scenario PU on the two retailers' profits. It could be observed that retailer R2 would choose newer green product b when $c_{m b}$ locates in the interval $(0.08,0.58)$ in which $\Pi_{\mathrm{R} 1}^{\mathrm{PU}^{*}}-\Pi_{\mathrm{R} 1}^{\mathrm{PP}^{*}}$ is always negative.

In the scenario when retailer R1 chooses to sell the newer green product $\mathrm{b}$, there are two cases to consider on the Pareto improvement in profits. We first survey the case when R2 is not active to sell the new product and then we have:

Proposition 5.2. In scenario UP, when $R 1$ adopts to sell product $b$ and $R 2$ does not, we have three cases to consider:

(1) If $0<\eta \leq \frac{49}{32}$, a Pareto improvement on both retailers $R 1$ and R2's profits could not be achieved.

(2) If $\eta>\frac{49}{32}$, a Pareto improvement on both retailers $R 1$ and R2's profits could be achieved when the condition $\tilde{c}_{m b, 3}<c_{m b}<\hat{c}_{m b, 3}$ is satisfied.

(3) If $\eta=\frac{49}{32}$, a Pareto improvement on both retailers $R 1$ and R2's profits could be achieved when $\frac{81\left(57 c_{m a}+8\right)}{2080}<$ $c_{m b}<\hat{c}_{m b, 3}$ is satisfied.

$$
\text { Here, } \hat{c}_{m b, 3}=-\frac{(\eta+1)\left(2 \eta\left(-2 \sqrt{\frac{4 \eta+2}{\eta^{2}+\eta}}\left(c_{m a}-1\right)-7\right)-7 c_{m a}\right)}{14 \eta+7} \text {. }
$$




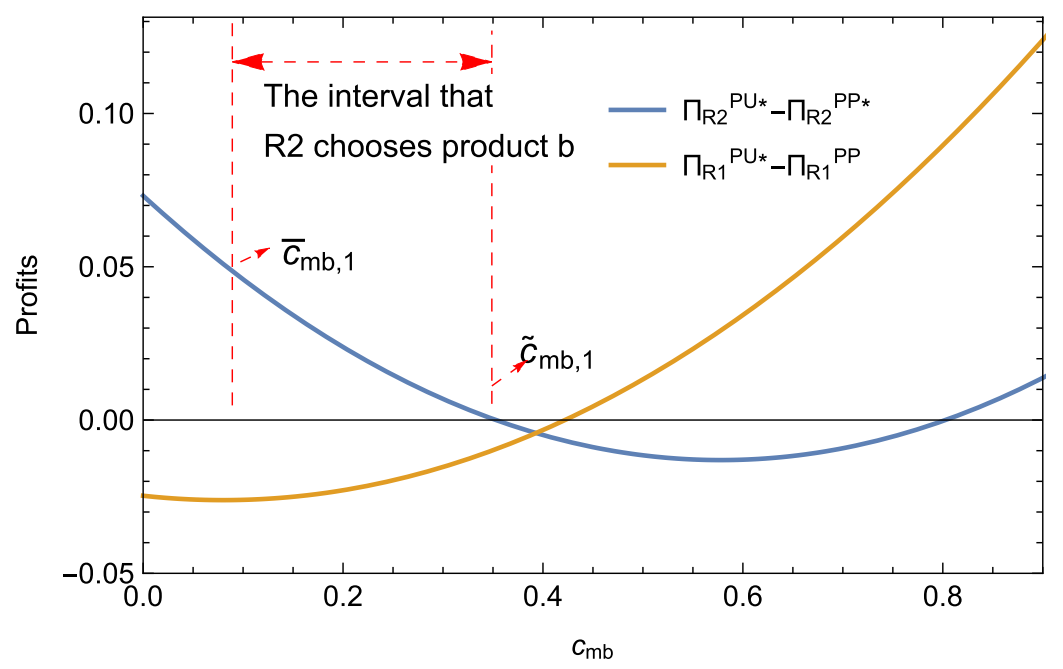

FiguRE 5. Retailers R1 and R2's profits improvement in scenario PU (with $c_{m a}=0.2$ and $\eta=0.2$ ).

In Proposition 5.2, we demonstrate the conditions under which retailer R1's profit would improved when retailers $\mathrm{R} 1$ and $\mathrm{R} 2$ sequentially chooses to sell products $\mathrm{b}$ and $\mathrm{a}$. The above results are derived based on the premise that retailer $\mathrm{R} 2$ would choose product a (if $\Pi_{\mathrm{R} 2}^{\mathrm{UP}}-\Pi_{\mathrm{R} 2}^{\mathrm{UU}}>0$ ), that is, we should investigate whether the profits of $\mathrm{R} 1$ and $\mathrm{R} 2$ will be increased at the same time under three situations, i.e., $\left.0<\eta<\frac{2}{49}, \eta\right\rangle \frac{49}{32}$ and $\eta=\frac{49}{32}$ (see Prop. 4.4).

Results show that when the consumer preference for product $b$ is small enough, the two retailers could not achieve a profit improvement at the same time. In the case when $0<\eta \leq \frac{49}{32}$, retailer R2 would choose product a if and only if $\bar{c}_{m b, 3}<c_{m b}<\bar{c}_{m b, 4}$ is satisfied (if $\frac{2}{49} \leq \eta<\frac{49}{32}$, R2 would choose to sell the newer green generation product all the time). However, the profit increment of retailer $\mathrm{R} 1\left(\Pi_{\mathrm{R} 1}^{\mathrm{UP}}-\Pi_{\mathrm{R} 1}^{\mathrm{PP}}\right)$ is functional concave with respect to $c_{m b}$ and has no root for $\Pi_{\mathrm{R} 1}^{\mathrm{UP}}-\Pi_{\mathrm{R} 1}^{\mathrm{PP}}=0$ in the interval $\bar{c}_{m b, 3}<c_{m b}<\bar{c}_{m b, 4}$, and therefore $\Pi_{\mathrm{R} 1}^{\mathrm{UP}^{*}}-\Pi_{\mathrm{R} 1}^{\mathrm{PP}}<0$ exists all the time when $0<\eta \leq \frac{49}{32}$. In the case when $\eta>\frac{49}{32}, \Pi_{\mathrm{R} 1}^{\mathrm{UP}^{*}}-\Pi_{\mathrm{R} 1}^{\mathrm{PP}}$ and $\Pi_{\mathrm{R} 1}^{\mathrm{UP}^{*}}-\Pi_{\mathrm{R} 1}^{\mathrm{PP}^{*}}$ are both convex with $c_{m b}$, and retailer $\mathrm{R} 2$ would choose product a $\left(\Pi_{\mathrm{R} 2}^{\mathrm{UP}^{*}}-\Pi_{\mathrm{R} 2}^{\mathrm{UU}}>0\right)$ if and only if $\tilde{c}_{m b, 3}<c_{m b}<\bar{c}_{m b, 4}$ is satisfied, it could be proved that the smaller root $\left(\hat{c}_{m b, 3}\right)$ of $\Pi_{\mathrm{R} 1}^{\mathrm{UP}^{*}}-\Pi_{\mathrm{R} 1}^{\mathrm{PP}^{*}}=0$ is smaller than $\tilde{c}_{m b, 3}$, then there exists a Pareto interval in which both R1 and R2's profits are improved. This could be explained that when consumer preference $(\eta)$ for the green product $\mathrm{b}$ is too small $\left(0<\eta \leq \frac{49}{32}\right)$, retailer R1 would not choose the new product because of the possible low revenue margin. For the case when $\eta>\frac{49}{32}$, we use a numerical example to show the variation of profits (see Figure 6).

In the scenario when both retailers R1 and R2 choose to sell the new generation product, there are two cases to be considered on studying the Pareto improvement in profits brought by green product introduction.

Proposition 5.3. In scenario UU, we have three cases to consider:

(1) If $\frac{1}{98}(\sqrt{2417}-45)<\eta<\frac{49}{32}$, when $\bar{c}_{m b, 3}<c_{m b}<\hat{c}_{m b, 3}$ is satisfied, a Pareto improvement on both retailers R1 and R2's profits could be achieved.

(2) If $\eta>\frac{49}{32}$, when $\bar{c}_{m b, 3}<c_{m b}<\tilde{c}_{m b, 3}$ is satisfied, a Pareto improvement on both retailers $R 1$ and R2's profits could be achieved.

(3) If $\eta=\frac{49}{32}$, when $\bar{c}_{m b, 3}<c_{m b}<\frac{81\left(57 c_{m a}+8\right)}{2080}$ is satisfied, a Pareto improvement on both retailers $R 1$ and R2's profits could be achieved.

Here, the closed forms of $\hat{c}_{m b, 3}, \tilde{c}_{m b, 3}$ and $\bar{c}_{m b, 3}$ could be found in the above propositions. 


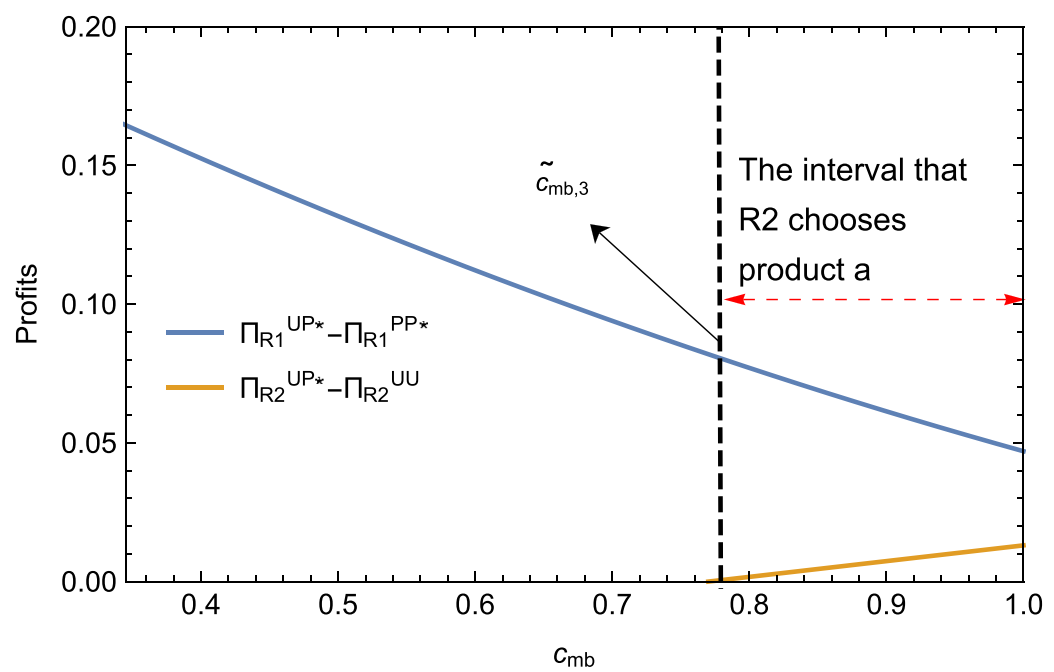

FiguRE 6. Retailers R1 and R2's profits improvement in scenario UP (with $c_{m a}=0.2$ and $\eta=\frac{50}{32}$ ).

In Proposition 5.3, we show that as long as $\eta$ is larger than the threshold $\frac{1}{98}(\sqrt{2417}-45)$, there exist possibilities that both retailers R1 and R2 could achieve Pareto improvement in profits. The findings in this proposition is not in accordance with Proposition 5.2, in which there is no Pareto when $\eta \leq \frac{49}{32}$. Note that in case (3), when $\eta=\frac{49}{32}$, the non-negative lower limits is negative and thus the condition $\bar{c}_{m b, 3}<c_{m b}<\frac{81\left(57 c_{m a}+8\right)}{2080}$ could be simplified as $0<c_{m b}<\frac{81\left(57 c_{m a}+8\right)}{2080}$. For the case when $\eta=\frac{49}{32}$, we use a numerical example to illustrate the varies of profits (see Figure 7).

The above results indicate that although the introduction of newer green products might benefit R2, but in specific cases, the profit of retailer R1 will not necessarily increase. As a Stackelberg leader, how should retailer R1 make her final product strategy? In Section 4, we focus on analysing R2's optimal decision (prices or quantities) with the complete information of $\mathrm{R} 1$, and we give the strategic product selection of $\mathrm{R} 2$ in different cases identified by thresholds of cost and consumer preference. It is noted that R1, as a decision forerunner, can observe the optimal response strategy of R2. An interesting question is how the first-mover R1 makes the optimal product decision.

\section{The LEADER RETAILER R1's STRATEgiC CHOICE}

To ensure retailer R1 could make her trade-off from the two product strategies (and retailer R2 would respond and take part in the supply chain when R1 select either of the two products), all the analysis are working within the interval that $\bar{c}_{m b, 1}<c_{m b}<\bar{c}_{m b, 4}$ (Here we have $\bar{c}_{m b, 3}<\bar{c}_{m b, 1}<\bar{c}_{m b, 4}<\bar{c}_{m b, 2}$ ). See the overlapped interval described in Figure 8.

By observing the strategic responses of retailer R2 in different cost threshold intervals (demonstrated by Props. 4.2 and 4.4), we give retailer R1's ex ante decisions as follows:

Proposition 6.1. We have four scenarios to consider:

(1) If $0<\eta \leq \frac{2}{49}$, when $\bar{c}_{m b, 1}<c_{m b}<\bar{c}_{m b, 4}$, R2 would follow R1's product choice all the time, and R1 would choose product a if $\Pi_{\mathrm{R} 1}^{\mathrm{PP}}>\Pi_{\mathrm{R} 1}^{\mathrm{UU}^{*}}$, and green product $b$ if $\Pi_{\mathrm{R} 1}^{\mathrm{PP}^{*}} \leq \Pi_{\mathrm{R} 1}^{\mathrm{UU}^{*}}$.

(2) If $\frac{2}{49}<\eta<\frac{49}{32}$, when $\bar{c}_{m b, 1}<c_{m b}<\tilde{c}_{m b, 1}, R 1$ would choose green product $b$ if $\Pi_{\mathrm{R} 1}^{\mathrm{UU}^{*}}>\Pi_{\mathrm{R} 1}^{\mathrm{PU}}$, and product a if $\Pi_{\mathrm{R} 1}^{\mathrm{UU}^{*}} \leq \Pi_{\mathrm{R} 1}^{\mathrm{PU}}$; when $\tilde{c}_{m b, 1}<c_{m b}<\bar{c}_{m b, 4}$, R1 would choose green product $b$ if $\Pi_{\mathrm{R} 1}^{\mathrm{PP}}<\Pi_{\mathrm{R} 1}^{\mathrm{UU}^{*}}$, and product a if $\Pi_{\mathrm{R} 1}^{\mathrm{PP}^{*}} \geq \Pi_{\mathrm{R} 1}^{\mathrm{UU}^{*}}$. 


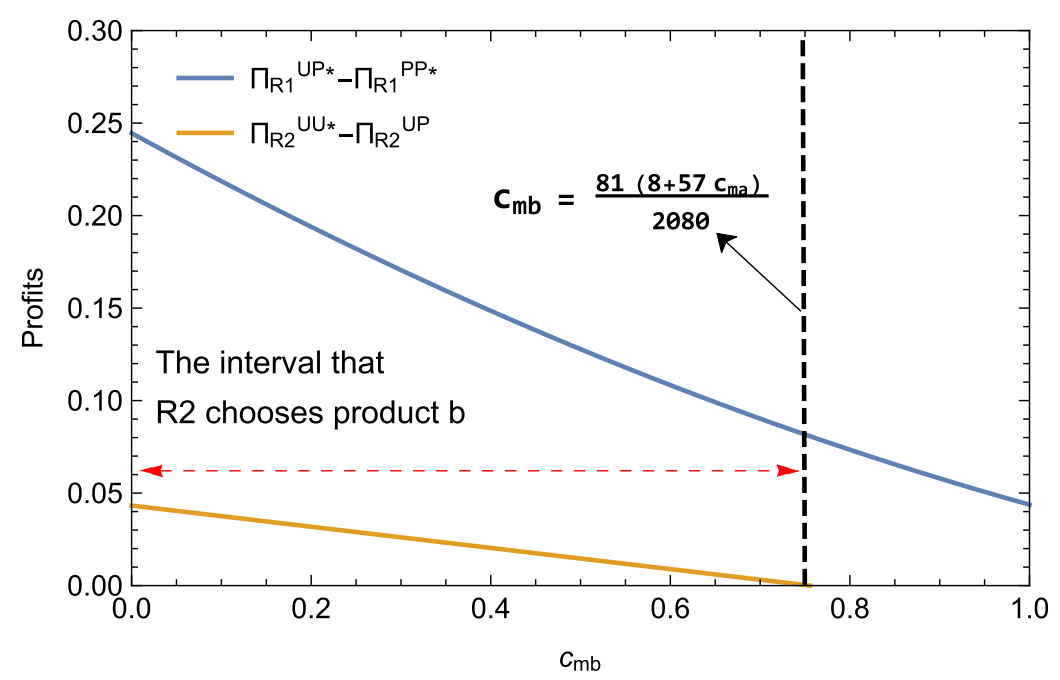

FiguRE 7. Retailers R1 and R2's profits improvement in scenario UU (with $c_{m a}=0.2$ and $\eta=\frac{49}{32}$ ).

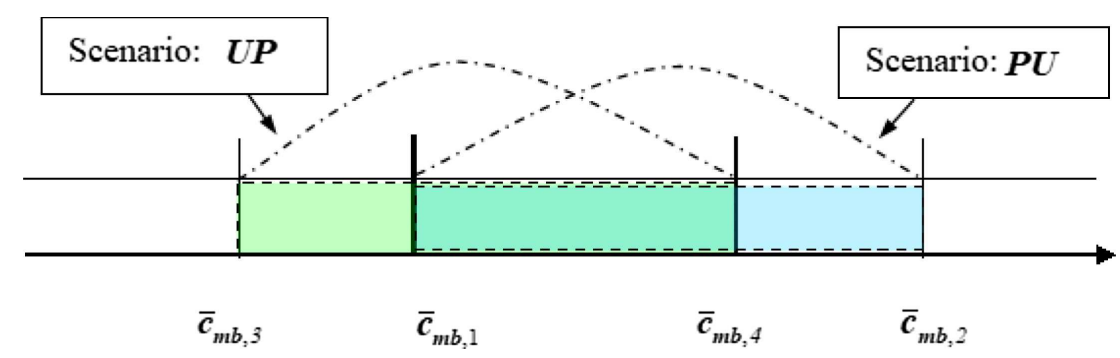

FiguRE 8. The non-negativity intervals of the four scenarios.

(3) If $\eta=\frac{49}{32}$, when $\bar{c}_{m b, 1}<c_{m b}<\frac{81\left(57 c_{m a}+8\right)}{2080}$, R1 would choose green product $b$ if $\Pi_{\mathrm{R} 1}^{\mathrm{PU}^{*}}<\Pi_{\mathrm{R} 1}^{\mathrm{UU}^{*}}$, and product a if $\Pi_{\mathrm{R} 1}^{\mathrm{PU}^{*}} \geq \Pi_{\mathrm{R} 1}^{\mathrm{UU}^{*}}$; when $\frac{81\left(57 c_{m a}+8\right)}{2080}<c_{m b}<\tilde{c}_{m b, 1}$, R1 would choose product a if $\Pi_{\mathrm{R} 1}^{\mathrm{PU}}>\Pi_{\mathrm{R} 1}^{\mathrm{UP}}$, and green product $b$ if $\Pi_{\mathrm{R} 1}^{\mathrm{PU}} \leq \Pi_{\mathrm{R} 1}^{\mathrm{UP}}$; when $\tilde{c}_{m b, 1}<c_{m b}<\bar{c}_{m b, 4}$, R1 would choose green product $b$ if $\Pi_{\mathrm{R} 1}^{\mathrm{PP}^{*}}<\Pi_{\mathrm{R} 1}^{\mathrm{UP}^{*}}$, and product a if $\Pi_{\mathrm{R} 1}^{\mathrm{PP}^{*}} \geq \Pi_{\mathrm{R} 1}^{\mathrm{UP}}$.

(4) If $\eta>\frac{49}{32}$, when $\bar{c}_{m b, 1}<c_{m b}<\tilde{c}_{m b, 3}$, R1 would choose product a if $\Pi_{\mathrm{R} 1}^{\mathrm{PP}^{*}}>\Pi_{\mathrm{R} 1}^{\mathrm{UU}^{*}}$, and green product $b$ if $\Pi_{\mathrm{R} 1}^{\mathrm{PP}^{*}} \leq \Pi_{\mathrm{R} 1}^{\mathrm{UU}}$; when $\tilde{c}_{m b, 3}<c_{m b}<\tilde{c}_{m b, 1}, R 1$ would choose product a if $\Pi_{\mathrm{R} 1}^{\mathrm{PU}}>\Pi_{\mathrm{R} 1}^{\mathrm{UP}^{*}}$, and green product $b$ if $\Pi_{\mathrm{R} 1}^{\mathrm{PU}} \leq \Pi_{\mathrm{R} 1}^{\mathrm{UP}}$; when $\tilde{c}_{m b, 1}<c_{m b}<\bar{c}_{m b, 4}$, R1 would choose product a if $\Pi_{\mathrm{R} 1}^{\mathrm{PP}^{*}}>\Pi_{\mathrm{R} 1}^{\mathrm{UP}}$, and green product $b$ if $\Pi_{\mathrm{R} 1}^{\mathrm{PP}} \leq \Pi_{\mathrm{R} 1}^{\mathrm{UP}}$.

In Proposition 6.1, we show how the leader retailer makes optimal product selection strategy in four scenarios. In case (1), if $0<\eta \leq \frac{2}{49}$, we show that there exist two possible sub-cases: (1) retailer R2 has to choose product $\mathrm{a}$ if retailer $\mathrm{R} 1$ chooses product a; $(2)$ retailer $\mathrm{R} 2$ could choose green product $\mathrm{b}$ (when $\bar{c}_{m b, 3}<c_{m b}<\tilde{c}_{m b, 3}$ ) or a (when $\tilde{c}_{m b, 3}<c_{m b}<\bar{c}_{m b, 4}$ ) if retailer R1 chooses product b. Because $\tilde{c}_{m b, 3}>\bar{c}_{m b, 4}$ in this case, then in R1's optional interval, i.e., $\bar{c}_{m b, 1}<c_{m b}<\bar{c}_{m b, 4}$, R2 would follow R1's product strategy no matter R1 chooses products a or b. Thus, R1 would choose product a if $\Pi_{\mathrm{R} 1}^{\mathrm{PP}}>\Pi_{\mathrm{R} 1}^{\mathrm{UU}^{*}}$, and product $\mathrm{b}$ if $\Pi_{\mathrm{R} 1}^{\mathrm{PP}} \leq \Pi_{\mathrm{R} 1}^{\mathrm{UU}}$. Among these two cases, retailer R1 could forecast retailer R2's strategy and induce R2 to follow or not to follow her product strategy since choosing a wrong product strategy would incur a lower profit for R2 (as a rational 
profit-maximizer) (see Props. 4.2 and 4.4). Thus, as the leader, retailer R1's trade-off on product strategy can be obtained by comparing her profit functions under different choices.

Note that in Proposition 6.1, we only show how retailer R1 should make her product selection strategies in specific situations (of $\eta$ ) and specific intervals (of $c_{m b}$ ), that is to say, we do not give the correspondent detailed thresholds. It could be observed that the final decisions of $\mathrm{R} 1$ lies on the profit differences, i.e., $\Pi_{\mathrm{R} 1}^{\mathrm{UP}^{*}}-\Pi_{\mathrm{R} 1}^{\mathrm{PP}^{*}}$, $\Pi_{\mathrm{R} 1}^{\mathrm{PP}^{*}}-\Pi_{\mathrm{R} 1}^{\mathrm{UU}^{*}}, \Pi_{\mathrm{R} 1}^{\mathrm{PU}^{*}}-\Pi_{\mathrm{R} 1}^{\mathrm{UP}}$ and $\Pi_{\mathrm{R} 1}^{\mathrm{PU}^{*}}-\Pi_{\mathrm{R} 1}^{\mathrm{UU}^{*}}$. For the first two comparisons, we have given the threshold points in Section 5. Here, we show the results of the last three profit comparisons.

Lemma 6.2. - For $\Pi_{\mathrm{R} 1}^{\mathrm{PP}}$ and $\Pi_{\mathrm{R} 1}^{\mathrm{UU}^{*}}$ : we have $\Pi_{\mathrm{R} 1}^{\mathrm{PP}^{*}}-\Pi_{\mathrm{R} 1}^{\mathrm{UU}^{*}}>0$ if $\hat{c}_{m b, 5}<c_{m b}<\hat{c}_{m b, 6}$; $\Pi_{\mathrm{R} 1}^{\mathrm{PP}^{*}}-\Pi_{\mathrm{R} 1}^{\mathrm{UU}^{*}} \leq 0$ if $c_{m b} \leq \hat{c}_{m b, 5}$ or $c_{m b} \geq \hat{c}_{m b, 6}$.

- For $\Pi_{\mathrm{R} 1}^{\mathrm{PU}}$ and $\Pi_{\mathrm{R} 1}^{\mathrm{UU}}$ :

(1) when $\frac{1}{190}(6 \sqrt{131}-59)<\eta<\frac{49}{380}$, we have $\Pi_{\mathrm{R} 1}^{\mathrm{PU}^{*}}-\Pi_{\mathrm{R} 1}^{\mathrm{UU}^{*}}<0$ if $\hat{c}_{m b, 8}<c_{m b}<\hat{c}_{m b, 7} ; \Pi_{\mathrm{R} 1}^{\mathrm{PU}^{*}}-\Pi_{\mathrm{R} 1}^{\mathrm{UU}^{*}} \geq 0$ if $c_{m b} \leq \hat{c}_{m b, 8}$ or $c_{m b} \geq \hat{c}_{m b, 7}$

(2) when $\eta=\frac{49}{380}$, we have $\Pi_{\mathrm{R} 1}^{\mathrm{PU}^{*}}-\Pi_{\mathrm{R} 1}^{\mathrm{UU}}>0$ if $c_{m b}>\frac{10892739 c_{m a}-1152294}{8627900} ; \Pi_{\mathrm{R} 1}^{\mathrm{PU}}-\Pi_{\mathrm{R} 1}^{\mathrm{UU}^{*}} \leq 0$ if $c_{m b} \leq$ $10892739 c_{m a}-1152294$;

(3) when $\eta>\frac{49}{380}$, we have $\Pi_{\mathrm{R} 1}^{\mathrm{PU}^{*}}-\Pi_{\mathrm{R} 1}^{\mathrm{UU}}>0$ if $\hat{c}_{m b, 7}<c_{m b}<\hat{c}_{m b, 8} ; \Pi_{\mathrm{R} 1}^{\mathrm{PU}^{*}}-\Pi_{\mathrm{R} 1}^{\mathrm{UU}^{*}} \leq 0$ if $c_{m b} \leq \hat{c}_{m b, 7}$ or $c_{m b} \geq \hat{c}_{m b, 8}$

(4) specially, $\Pi_{\mathrm{R} 1}^{\mathrm{PU}^{*}}-\Pi_{\mathrm{R} 1}^{\mathrm{UU}^{*}}>0$ all the time when $\frac{2}{49}<c_{m b}<\frac{1}{190}(6 \sqrt{131}-59)^{5}$;

- For $\Pi_{\mathrm{R} 1}^{\mathrm{PU}^{*}}$ and $\Pi_{\mathrm{R} 1}^{\mathrm{UP}^{*}}:$ we have $\Pi_{\mathrm{R} 1}^{\mathrm{PU}^{*}}-\Pi_{\mathrm{R} 1}^{\mathrm{UP}^{*}}>0$ if $\hat{c}_{m b, 9}<c_{m b}<\hat{c}_{m b, 10} ; \Pi_{\mathrm{R} 1}^{\mathrm{PU}^{*}}-\Pi_{\mathrm{R} 1}^{\mathrm{UP}^{*}} \leq 0$ if $c_{m b} \leq \hat{c}_{m b, 9}$ or $c_{m b} \geq \hat{c}_{m b, 10}$.

$$
\begin{gathered}
\text { Here, } \quad \hat{c}_{m b, 5}=-(\eta+1)\left(\sqrt{\frac{\left(c_{m a}-1\right)^{2}}{\eta+1}}-1\right), \quad \hat{c}_{m b, 6}=(\eta+1)\left(\sqrt{\frac{\left(c_{m a}-1\right)^{2}}{\eta+1}}+1\right), \quad \hat{c}_{m b, 7}= \\
\frac{(\eta+1)\left(2 \eta\left(14 \sqrt{\frac{(4 \eta(95 \eta+59)-13)\left(c_{m a}-1\right)^{2}}{\eta}}+380 \eta+141\right)-49 c_{m a}\right)}{\eta}, \hat{c}_{m b, 8}=\frac{(\eta+1)\left(2 \eta\left(-14 \sqrt{\frac{(4 \eta(95 \eta+59)-13)\left(c_{m a}-1\right)^{2}}{\eta}}+380 \eta+141\right)-49 c_{m a}\right)}{(2 \eta+1)(380 \eta-49)}, \\
\hat{c}_{m b, 9}=\frac{(\eta+1)\left(-\sqrt{\frac{(8 \eta+5)^{2}\left(c_{m a}-1\right)^{2}}{\eta+1}}+8 \eta+5\right)}{8 \eta+5} \text { and } \hat{c}_{m b, 10}=\frac{(\eta+1)\left(\sqrt{\frac{(8 \eta+5)^{2}\left(c_{m a}-1\right)^{2}}{\eta+1}}+8 \eta+5\right)}{8 \eta+5} .
\end{gathered}
$$

Note that in the intervals considered in Proposition 6.1, R1 could be freely selected from product a or b. However, outside this range, R2 will not join the supply chain (see Props. 4.2 and 4.4), and in such conditions $\mathrm{R} 1$ has no choice but to select one or none of the two product strategies. By combining the results demonstrated in Proposition 6.1 and Lemma 6.2, we use Table 6 to summarize and show the detailed product strategies of R1.

In Table 6, we demonstrate R1's decisions with thresholds defined in Lemma 6.2 and Proposition 6.1 in four cases. Specially, in case (2) where $\frac{2}{49}<\eta<\frac{49}{32}$, we further subdivide the results of optimal strategy in four scenarios. Because there exist more than 16 intervals that defining the optimal product decisions, it is complicated to give further simplified results and we decide to express the decision intervals in the form of set defined by thresholds of $\eta$ and $c_{m b}$.

To better understand how retailers R1 and R2 jointly obtain their optimal product and pricing strategies, we use the following numerical example to show retailer R1's decision zone.

For cases (1) and (4) in Proposition 6.1, we use Figures 9 and 10 (with $c_{m a}=0.25$ ) to show R1's decision zone. These two cases include the conditions where $\eta$ is extremely small $\left(0<\eta \leq \frac{2}{49}\right)$ or relatively large $\left(\eta>\frac{49}{32}\right)$. It could be found that retailer R1 could make her strategic product choice between the two shaded areas (the red and blue areas respectively denote the choice of products a and $\mathrm{b}$ ). Results show that when $c_{m b}$ is large enough (or small enough), R1 would select product a (or b). For case $(3)\left(\eta=\frac{49}{32}\right)$ and the special case $\left(\eta=\frac{49}{380}\right)$ of case (2), the decision zone of R1 would change when product a's unit cost $c_{m a}$ varies. We choose to show the case of $\eta=\frac{49}{32}$ in Figure 11. It could be found that R1 would choose product $\mathrm{b}$ all the time when $\eta=\frac{49}{32}$, and all the thresholds that define R1's choices of product a are not distributed in the possible scope $\left(0<c_{m a}<1\right)$

\footnotetext{
${ }^{5} \hat{c}_{m b, 7}<\hat{c}_{m b, 8}$ if $\eta>\frac{49}{380}$ and $\hat{c}_{m b, 7}>\hat{c}_{m b, 8}$ if $\eta<\frac{49}{380}$. When $\eta=\frac{49}{380}$, there is only one root $\left(c_{m b}=\frac{10892739 c_{m a}-1152294}{8627900}\right)$ for $\Pi_{\mathrm{R} 1}^{\mathrm{PU}^{*}}-\Pi_{\mathrm{R} 1}^{\mathrm{UU}^{*}}=0$.
} 
TABle 6. Product strategies of retailer R1.

\begin{tabular}{lll}
\hline \hline Cases & Product a & Product b \\
\hline $0<\eta \leq \frac{2}{49}$ & $\Phi_{1} \bigcap \Psi_{11}$ & $\Phi_{1} \bigcap \Psi_{12}$ \\
\hline & If $\frac{2}{49}<\eta<\frac{6 \sqrt{131}-59}{190}$, & If $\frac{2}{49}<\eta<\frac{6 \sqrt{131}-59}{190}$, \\
& $\Phi_{2}$ or $\Phi_{3} \bigcap \Psi_{11}$ & $\Phi_{3} \bigcap \Psi_{12}$ \\
$\frac{2}{49}<\eta<\frac{49}{32}$ & If $\frac{6 \sqrt{131}-59}{190}<\eta<\frac{49}{380}$, & If $\frac{6 \sqrt{131}-59}{190}<\eta<\frac{49}{380}$, \\
& $\Phi_{2} \bigcap \Psi_{21}$ or $\Phi_{3} \bigcap \Psi_{11}$ & $\Phi_{2} \bigcap \Psi_{22}$ or $\Phi_{3} \bigcap \Psi_{12}$ \\
& If $\eta=\frac{49}{380}$, & If $\eta=\frac{49}{380}$, \\
& $\Phi_{2} \bigcap \Psi_{31}$ or $\Phi_{3} \bigcap \Psi_{11}$ & $\Phi_{2} \bigcap \Psi_{32}$ or $\Phi_{3} \bigcap \Psi_{12}$ \\
& If $\frac{49}{380}<\eta<\frac{49}{32}$, & If $\frac{49}{380}<\eta<\frac{49}{32}$, \\
& $\Phi_{2} \bigcap \Psi_{41}$ or $\Phi_{3} \bigcap \Psi_{11}$ & $\Phi_{2} \bigcap \Psi_{42}$ or $\Phi_{3} \bigcap \Psi_{12}$ \\
\hline$\eta \frac{49}{32}$ & $\Phi_{4} \bigcap \Psi_{41}$ & $\Phi_{4} \bigcap \Psi_{42}$ \\
& or $\Phi_{5} \bigcap \Psi_{51}$ or $\Phi_{3} \bigcap \Psi_{61}$ & or $\Phi_{5} \bigcap \Psi_{52}$ or $\Phi_{3} \bigcap \Psi_{62}$ \\
\hline$\eta \frac{49}{32}$ & $\Phi_{6} \bigcap \Psi_{11}$ & $\Phi_{6} \bigcap \Psi_{12}$ \\
& or $\Phi_{7} \bigcap \Psi_{51}$ or $\Phi_{3} \bigcap \Psi_{61}$ & or $\Phi_{7} \bigcap \Psi_{52}$ or $\Phi_{3} \bigcap \Psi_{62}$ \\
\hline
\end{tabular}

Notes. Here, $\Phi_{1} \equiv\left\{\bar{c}_{m b, 1}<c_{m b}<\bar{c}_{m b, 4}\right\}, \Phi_{2} \equiv\left\{\bar{c}_{m b, 1}<c_{m b}<\tilde{c}_{m b, 1}\right\}, \Phi_{3} \equiv\left\{\tilde{c}_{m b, 1}<c_{m b}<\bar{c}_{m b, 4}\right\}, \Phi_{4} \equiv\left\{\bar{c}_{m b, 1}<\right.$ $\left.c_{m b}<\frac{81\left(57 c_{m a}+8\right)}{2080}\right\}, \Phi_{5} \equiv\left\{\frac{81\left(57 c_{m a}+8\right)}{2080}<c_{m b}<\tilde{c}_{m b, 1}\right\}, \Phi_{6} \equiv\left\{\bar{c}_{m b, 1}<c_{m b}<\tilde{c}_{m b, 3}\right\}, \Phi_{7} \equiv\left\{\tilde{c}_{m b, 3}<c_{m b}<\tilde{c}_{m b, 1}\right\}$ and $\Psi_{11} \equiv\left\{\hat{c}_{m b, 5}<c_{m b}<\hat{c}_{m b, 6}\right\}, \Psi_{12} \equiv\left\{c_{m b} \leq \hat{c}_{m b, 5} \bigcup c_{m b} \geq \hat{c}_{m b, 6}\right\}, \Psi_{21} \equiv\left\{c_{m b} \leq \hat{c}_{m b, 8} \bigcup c_{m b} \geq \hat{c}_{m b, 7}\right\}, \Psi_{22} \equiv\left\{\hat{c}_{m b, 8}<\right.$ $\left.c_{m b}<\hat{c}_{m b, 7}\right\}, \Psi_{31} \equiv\left\{c_{m b} \geq \frac{10892739 c_{m a}-1152294}{8627900}\right\}, \Psi_{32} \equiv\left\{c_{m b}<\frac{10892739 c_{m a}-1152294}{8627900}\right\}, \Psi_{41} \equiv\left\{\hat{c}_{m b, 7}<c_{m b}<\hat{c}_{m b, 8}\right\}$, $\Psi_{42} \equiv\left\{c_{m b} \leq \hat{c}_{m b, 7} \bigcup c_{m b} \geq \hat{c}_{m b, 8}\right\}, \Psi_{51} \equiv\left\{\hat{c}_{m b, 9}<c_{m b}<\hat{c}_{m b, 10}\right\}, \Psi_{52} \equiv\left\{c_{m b} \leq \hat{c}_{m b, 9}\right.$ or $\left.c_{m b} \geq \hat{c}_{m b, 10}\right\} \Psi_{61} \equiv\left\{c_{m b} \leq\right.$ $\left.\hat{c}_{m b, 3} \bigcup c_{m b} \geq \hat{c}_{m b, 4}\right\}, \Psi_{62} \equiv\left\{\hat{c}_{m b, 3}<c_{m b}<\hat{c}_{m b, 4}\right\}$.

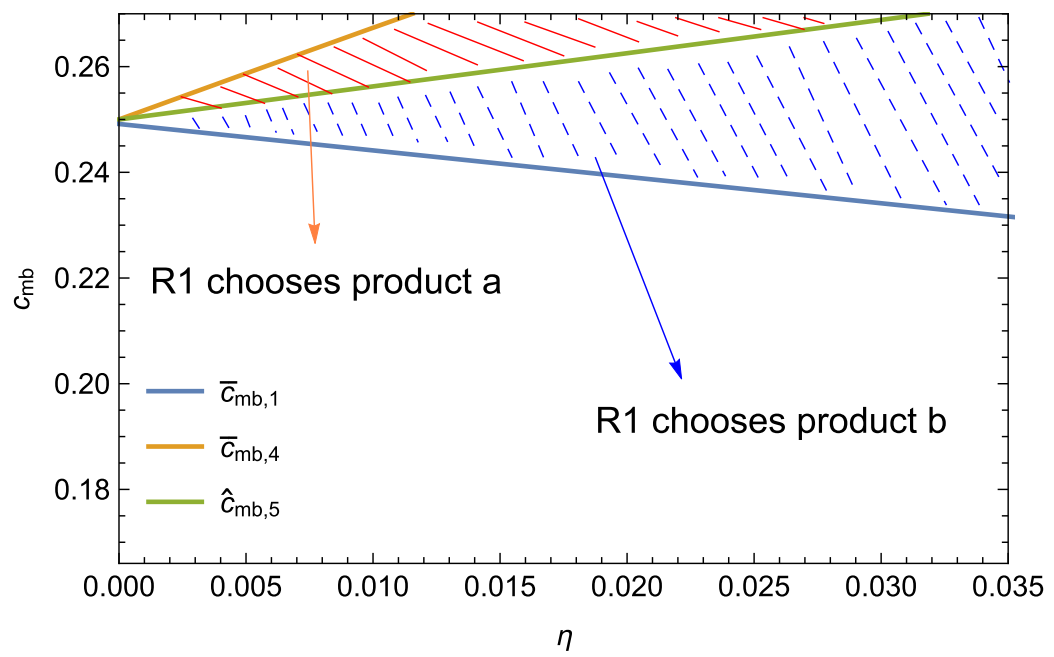

FiguRE 9. Retailers R1's strategic product choice when $0<\eta \leq \frac{2}{49}$ (with $c_{m a}=0.25$ ). 


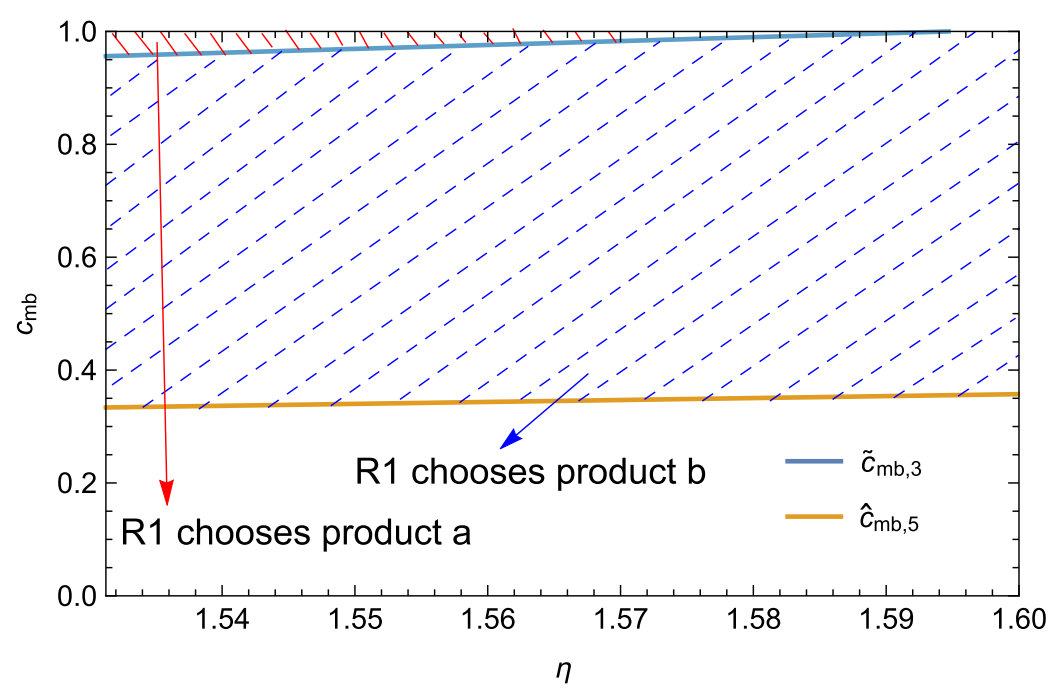

FiguRE 10. Retailers R1's strategic product choice when $\eta>\frac{49}{32}$ (with $c_{m a}=0.02$ ).

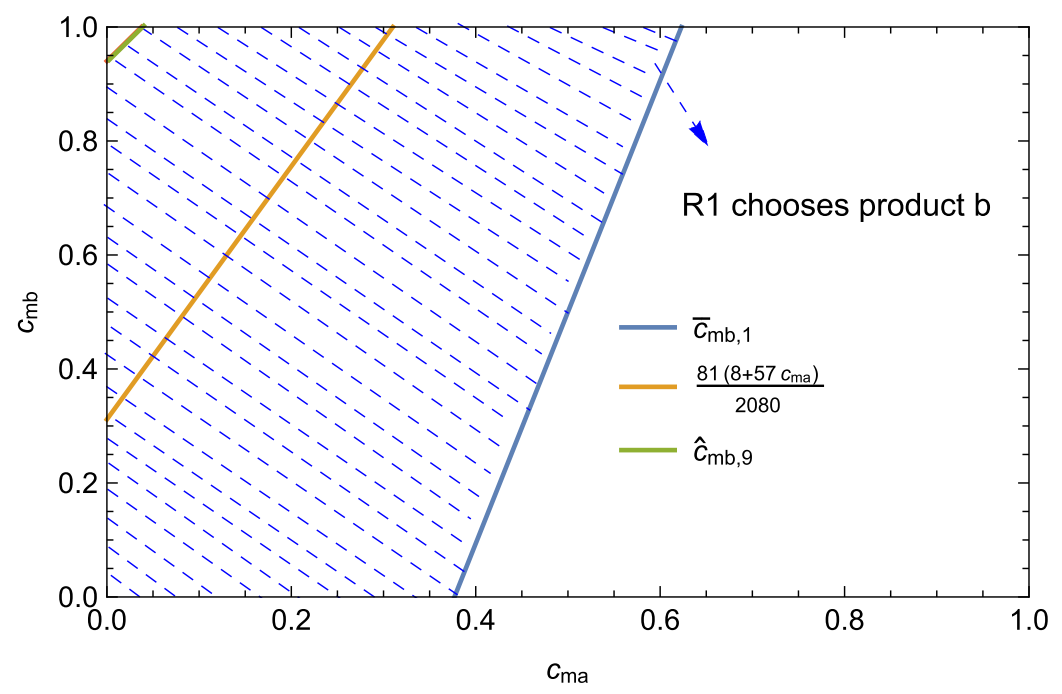

FiguRE 11. Retailers R1's strategic product choice when $\eta=\frac{49}{32}$.

in this figure. For case (2) when $\frac{2}{49}<\eta<\frac{49}{32}$, there are four sub-cases, we choose to study the sub-case when $\frac{49}{380}<\eta<\frac{49}{32}$ in Figure 12. We observe that in the "selectable" regions between the line $\bar{c}_{m b, 1}$ and $\bar{c}_{m b, 4}, \mathrm{R} 1$ would select product a if unit $\operatorname{cost} c_{m b}$ is high and consumers' green preference $\eta$ is low, and select green product $\mathrm{b}$ if $c_{m b}$ is low and $\eta$ is high.

\section{Conclusion}

The introduction of new products is a routine matter for many enterprises and it is also an important means to achieve the long-term profits. Under the pressure of environmental protection, industrial manufacturers have launched green value-added products to meet the environmental requirements from the government and 


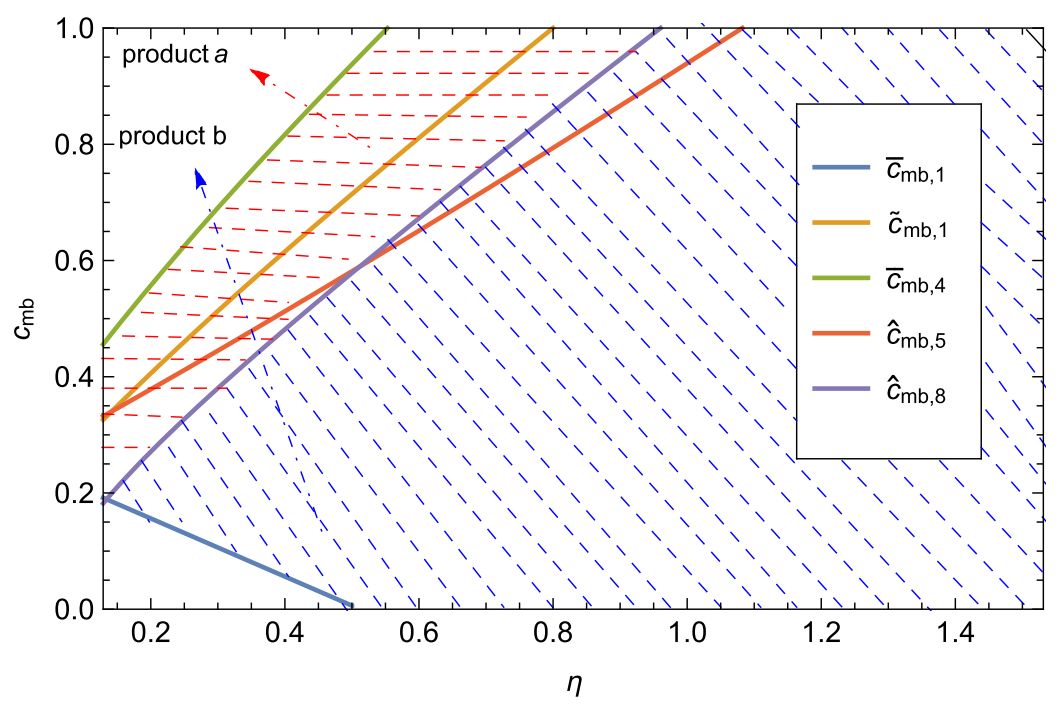

FigURE 12. Retailers R1's strategic product choice when $\frac{49}{380}<\eta<\frac{49}{32}$ (with $c_{m a}=0.25$ ).

consumers. Whether green products can enter the market successfully depends not only on the improvement of their own quality, but also on the market behavior of retail agents. As the supply chain middleman, retailers play a bridge role between the manufacturer and the consumers, and also plays an important role in distributing newer green products.

In this paper, we establish a two-tier supply chain system consisting of a single manufacturer and two competitive retailers (R1 and R2). The manufacturer sells older generation product a and newer green generation product b through two retailers. We study the product strategies of a strong retailer (R1) and a follow-up retailer (R2) with backward inductions to capture their dynamic behaviours. In the decision sequence, R2, as the follower of R1, does not necessarily imitate the product strategy of R1. He will make his own actions by observing R1's actions. Results show that in specific intervals, choosing a product strategy different from R1 to compete for prices would bring more profits to R2; and in other intervals, although choosing a imitative product strategy following R1 to compete for quantities could only obtain half of the profit of R1, it is still greater than the profit brought by price competition. At the same time, we obtain the Pareto intervals in which the introduction of green products can increase profits for both retailers. Results show that, in some cases, introducing green products would bring an increase in profit to R2 but a decrease in profit to R1. In other words, the response behavior of R2 might force R1, as the dominant player, to choose the older generation product. To this end, we derive the product selection strategies for $\mathrm{R} 1$ in different situations. The findings of this paper have important managerial significance for investigating whether green products can be successfully introduced into the market. Another contribution of this paper is to provide management enlightenment for supply chain members to select products under NPI, and to provide modeling techniques for such problems. If someone would like to use the model, important parameters including market size, production cost, and consumer preference should be predetermined. These parameters can be collected and processed in practice for related firms or managers.

Our paper have the following possible extensions: (1) We assume that each retailer can only sell one single product (which has been explained in Sect. 3). In practice, there might exist powerful enough retailers to sell both new and old products. (2) We assume that the manufacturer is not affected by environmental laws such as carbon regulation, it is worthwhile to survey to what extent the introduction of the green new product could reduce the manufacturer's environmental pressure. (3) We assume that the retailers lead the supply chain. In 
further research, it is necessary and worthwhile to analyze how the competing retailers make their product strategies when the manufacturer dominates the supply chain.

\section{Appendix A. Proof of Lemmas}

The solving procedures of Lemmas 4.1 and 4.3. Because scenario PU (PP) and UP (UU) can be proved similarly, we choose to prove PU and UP. Proof of Lemma 4.1: By using backward induction, we first derive the manufacturer's optimal wholesale price decisions. In Lemma 4.1 (scenario PU), both the two kinds of products are sold in the market. Given the retail margin decisions of the retailers, the demand functions are given as $Q_{\mathrm{R} 2}^{\mathrm{PU}}=1-\frac{\left(m_{b}^{\mathrm{PU}}+r_{b}^{\mathrm{PU}}\right)-\left(m_{a}^{\mathrm{PU}}+r_{a}^{\mathrm{PU}}\right)}{\eta}$ and $Q_{\mathrm{R} 1}^{\mathrm{PU}}=\frac{\left(m_{b}^{\mathrm{PU}}+r_{b}^{\mathrm{PU}}\right)-\left(m_{a}^{\mathrm{PU}}+r_{a}^{\mathrm{PU}}\right)}{\eta}-\left(m_{a}^{\mathrm{PU}}+r_{a}^{\mathrm{PU}}\right)$. It is direct to find that the manufacturer's profit function $\Pi_{M}^{\mathrm{PU}}\left(m_{a}^{\mathrm{PU}}, m_{b}^{\mathrm{PU}}\right)$ is jointly concave, and by solving the first order conditions we have:

$$
\begin{aligned}
& m_{b}^{\mathrm{PU}}\left(r_{a}^{\mathrm{PU}}, r_{b}^{\mathrm{PU}}\right)=\frac{1}{2}\left(-r_{b}^{\mathrm{PU}}+c_{m b}+\eta+1\right) \\
& m_{a}^{\mathrm{PU}}\left(r_{a}^{\mathrm{PU}}, r_{b}^{\mathrm{PU}}\right)=\frac{1}{2}\left(-r_{a}^{\mathrm{PU}}+c_{m a}+1\right) .
\end{aligned}
$$

Then, we turn to solve the follow-up retailer R2's optimal decision. Submitting $m_{b}\left(r_{a}, r_{b}\right)$ and $m_{a}\left(r_{a}, r_{b}\right)$ into R2's profit function, we have:

$$
\Pi_{\mathrm{R} 2}^{\mathrm{PU}}\left(r_{b}^{\mathrm{PU}} \mid r_{a}^{\mathrm{PU}}\right)=\frac{r_{b}^{\mathrm{PU}}\left(r_{a}^{\mathrm{PU}}-r_{b}^{\mathrm{PU}}+c_{m a}-c_{m b}+\eta\right)}{2 \eta} .
$$

Then R2's optimal response with given $r_{a}$ is

$$
r_{b}^{\mathrm{PU}}\left(r_{a}^{\mathrm{PU}}\right)=\frac{1}{2}\left(r_{a}^{\mathrm{PU}}+c_{m a}-c_{m b}+\eta\right)
$$

With these, we derive retailer R1's profit as:

$$
\Pi_{\mathrm{R} 1}^{\mathrm{PU}}\left(r_{a}^{\mathrm{PU}}\right)=-\frac{r_{a}^{\mathrm{PU}}\left(2 \eta r_{a}^{\mathrm{PU}}+r_{a}^{\mathrm{PU}}+(2 \eta+1) c_{m a}-c_{m b}-\eta\right)}{4 \eta}
$$

which is concave on $r_{a}$, and the first order condition yields that $r_{a}^{\mathrm{PU}^{*}}=\frac{\eta-2 \eta c_{m a}-c_{m a}+c_{m b}}{2(2 \eta+1)}$. Submitting the optimal result into the process variables, we have Lemma 4.1. In Lemma 4.3 (scenario UU), both the two retailers choose to sell product $b$. Then, they compete in quantities. The pricing function is therefore given as: $p_{b}^{\mathrm{UU}}=m_{b}^{\mathrm{UU}}+r_{b}^{\mathrm{UU}}=(\eta+1)\left(1-\left(Q_{\mathrm{R} 1}^{\mathrm{UU}}+Q_{\mathrm{R} 2}^{\mathrm{UU}}\right)\right)$. Still with backward induction, the manufacturer's profit $\Pi_{M}^{\mathrm{UU}}\left(m_{b}^{\mathrm{UU}}\right)$ can be expressed as:

$$
\Pi_{M}^{\mathrm{UU}}\left(m_{b}^{\mathrm{UU}}\right)=\frac{\left(c_{m b}-m_{b}^{\mathrm{UU}}\right)\left(m_{b}^{\mathrm{UU}}+r_{b}^{\mathrm{UU}}-\eta-1\right)}{\eta+1}
$$

which is concave on $m_{b}^{\mathrm{UU}}$, and the first order condition gives that

$$
m_{b}^{\mathrm{UU}}\left(r_{b}^{\mathrm{UU}}\right)=\frac{1}{2}\left(-r_{b}^{\mathrm{UU}}+c_{m b}+\eta+1\right) .
$$

Next, we show how the two retailers dynamically compete in quantities. First, we solve R2's response. R2's profit function $\Pi_{\mathrm{R} 2}^{\mathrm{UU}}\left(Q_{\mathrm{R} 2}^{\mathrm{UU}} \mid Q_{\mathrm{R} 1}^{\mathrm{UU}}\right)$ is solved as:

$$
\Pi_{\mathrm{R} 2}^{\mathrm{UU}}\left(Q_{\mathrm{R} 2}^{\mathrm{UU}} \mid Q_{\mathrm{R} 1}^{\mathrm{UU}}\right)=Q_{\mathrm{R} 2}^{\mathrm{UU}}\left(\frac{1}{2}\left(r_{b}-c_{m b}-\eta-1\right)+(\eta+1)\left(-Q_{\mathrm{R} 1}^{\mathrm{UU}}-Q_{\mathrm{R} 2}^{\mathrm{UU}}+1\right)\right) .
$$


This concave function gives that

$$
Q_{\mathrm{R} 2}^{\mathrm{UU}}\left(Q_{\mathrm{R} 1}^{\mathrm{UU}}\right)=\frac{r_{b}^{\mathrm{UU}}-c_{m b}+\eta-2 \eta Q_{\mathrm{R} 1}^{\mathrm{UU}}-2 Q_{\mathrm{R} 1}^{\mathrm{UU}}+1}{4(\eta+1)} .
$$

Then, as the supply chain leader, retailer R1 optimizes:

$$
\Pi_{\mathrm{R} 1}^{\mathrm{UU}}\left(Q_{\mathrm{R} 1}^{\mathrm{UU}}\right)=\frac{Q_{\mathrm{R} 1}^{\mathrm{UU}}}{4}\left(r_{b}^{\mathrm{UU}}-c_{m b}+\eta-2(\eta+1) Q_{\mathrm{R} 1}^{\mathrm{UU}}+1\right) .
$$

This concave function gives that $Q_{\mathrm{R} 1}^{\mathrm{UU}^{*}}=\frac{r_{b}^{\mathrm{UU}}-c_{m b}+\eta+1}{4(\eta+1)}$. Submitting the optimal result into the process variables above, we have Lemma 6.2.

\section{Appendix B. Proof of Propositions}

Proof of Proposition 4.2. Given that retailer R1 chooses to sell product a. Retailer R2's strategic choice can derived by solving the difference of $\Pi_{\mathrm{R} 2}^{\mathrm{PU}}$ and $\Pi_{\mathrm{R} 2}^{\mathrm{PP}}$. With the equilibrium prices and demands derived in Lemmas 4.1 and 4.3, we have:

$$
\Pi_{\mathrm{R} 2}^{\mathrm{PU}^{*}}-\Pi_{\mathrm{R} 2}^{\mathrm{PP}^{*}}=\frac{\left(c_{m a}+2 \eta c_{m a}-4 \eta c_{m b}-c_{m b}+4 \eta^{2}+3 \eta\right)^{2}}{32 \eta(2 \eta+1)^{2}}-\frac{1}{49}\left(c_{m a}-1\right)^{2} .
$$

By deriving the first and second order conditions of $\Pi_{\mathrm{R} 2}^{\mathrm{PU}^{*}}-\Pi_{\mathrm{R} 2}^{\mathrm{PP}}$, we have:

$$
\begin{aligned}
\frac{\partial\left(\Pi_{\mathrm{R} 2}^{\mathrm{PU}^{*}}-\Pi_{\mathrm{R} 2}^{\mathrm{PP}}\right)}{\partial c_{m b}} & =-\frac{(4 \eta+1)\left((2 \eta+1) c_{m a}-(4 \eta+1) c_{m b}+\eta(4 \eta+3)\right)}{16 \eta(2 \eta+1)^{2}} \\
\frac{\partial^{2}\left(\Pi_{\mathrm{R} 2}^{\mathrm{PU}^{*}}-\Pi_{\mathrm{R} 2}^{\mathrm{PP}^{*}}\right)}{\partial c_{m b}^{2}} & =\frac{(4 \eta+1)^{2}}{16 \eta(2 \eta+1)^{2}} .
\end{aligned}
$$

It is straightforward to have $\frac{\partial^{2}\left(\Pi_{\mathrm{R} 2}^{\mathrm{PU}}-\Pi_{\mathrm{R} 2}^{\mathrm{PP}}\right)}{\partial c_{m b}^{2}}>0$, which tells that $\Pi_{\mathrm{R} 2}^{\mathrm{PU}^{*}}-\Pi_{\mathrm{R} 2}^{\mathrm{PP}}$ is convex on $c_{m b}$. By solving $\Pi_{\mathrm{R} 2}^{\mathrm{PU}^{*}}-\Pi_{\mathrm{R} 2}^{\mathrm{PP}^{*}}=0$, we have two roots:

$$
\begin{gathered}
\tilde{c}_{m b, 1}=\frac{(2 \eta+1)\left(\frac{4 \sqrt{2}\left(c_{m a}-1\right)}{\sqrt{\frac{1}{\eta}}}+7 c_{m a}\right)+7 \eta(4 \eta+3)}{7(4 \eta+1)} \\
\tilde{c}_{m b, 2}=\frac{7 \eta(4 \eta+3)-(2 \eta+1)\left(\frac{4 \sqrt{2}\left(c_{m a}-1\right)}{\sqrt{\frac{1}{\eta}}}-7 c_{m a}\right)}{7(4 \eta+1)} .
\end{gathered}
$$

Thus, $\Pi_{\mathrm{R} 2}^{\mathrm{PU}^{*}}-\Pi_{\mathrm{R} 2}^{\mathrm{PP}}$ could be expressed as $\Pi_{\mathrm{R} 2}^{\mathrm{PU}}-\Pi_{\mathrm{R} 2}^{\mathrm{PP}}=\frac{(4 \eta+1)^{2}}{16 \eta(2 \eta+1)^{2}}\left(c_{m b}-\tilde{c}_{m b, 1}\right)\left(c_{m b}-\tilde{c}_{m b, 2}\right)$. Because $\tilde{c}_{m b, 2}-\tilde{c}_{m b, 1}=-\frac{8 \sqrt{2}(2 \eta+1)\left(c_{m a}-1\right)}{7 \sqrt{\frac{1}{\eta}}(4 \eta+1)}>0$, we have $\tilde{c}_{m b, 1}-\tilde{c}_{m b, 2}<0$. This yields that $\Pi_{\mathrm{R} 2}^{\mathrm{PU}^{*}}-\Pi_{\mathrm{R} 2}^{\mathrm{PP}^{*}}>0$ when $c_{m b}<\tilde{c}_{m b, 1}$ or $c_{m b}>\tilde{c}_{m b, 2}$ is satisfied. Note that the existence of scenario PU (The demands are positive) requires that $\bar{c}_{m b, 1}<c_{m b}<\bar{c}_{m b, 2}$. Here, $\bar{c}_{m b, 1}=(2 \eta+1) c_{m a}-\eta, \bar{c}_{m b, 2}=\frac{(2 \eta+1) c_{m a}+\eta(4 \eta+3)}{4 \eta+1}$. Because $\tilde{c}_{m b, 2}-$ $\bar{c}_{m b, 2}=-\frac{4 \sqrt{2}(2 \eta+1)\left(c_{m a}-1\right)}{7 \sqrt{\frac{1}{\eta}}(4 \eta+1)}>0$, therefore, the sign of $\Pi_{\mathrm{R} 2}^{\mathrm{PU}^{*}}-\Pi_{\mathrm{R} 2}^{\mathrm{PP}}{ }^{*}$ depends on the difference of $\bar{c}_{m b, 1}$ and $\tilde{c}_{m b, 1}$. We therefore have two cases to consider: (1) If $\eta>\frac{2}{49}$, we have $\bar{c}_{m b, 1}<\tilde{c}_{m b, 1}$, thus when $\bar{c}_{m b, 1}<c_{m b}<\tilde{c}_{m b, 1}$, retailer R2 would choose to sell the newER green product b; when $\tilde{c}_{m b, 1}<c_{m b}<\bar{c}_{m b, 2}$, retailer R2 would choose to sell the older generation product a. (2) If $\eta \leq \frac{2}{49}$, we have $\bar{c}_{m b, 1} \geq \tilde{c}_{m b, 1}$, thus $\Pi_{\mathrm{R} 2}^{\mathrm{P}}{ }^{*}-\Pi_{\mathrm{R} 2}^{\mathrm{PP}^{*}}<0$ all the time and retailer $\mathrm{R} 2$ should choose to sell the older generation product a. 
Proof of Proposition 4.4. Given that retailer R1 choose to sell product b. Retailer R2's strategic choice can derived by solving the difference of $\Pi_{\mathrm{R} 2}^{\mathrm{UP}}{ }^{*}$ and $\Pi_{\mathrm{R} 2}^{\mathrm{UU}}$. With the equilibrium prices and demands derived in Lemma 4.3, we have:

$$
\Pi_{\mathrm{R} 2}^{\mathrm{UU}^{*}}-\Pi_{\mathrm{R} 2}^{\mathrm{UP}^{*}}=\frac{\left(1-c_{m b}+\eta\right)^{2}}{49(\eta+1)}-\frac{\left((2 \eta+1) c_{m b}-\left(4 \eta^{2}+5 \eta+1\right) c_{m a}+2 \eta(\eta+1)\right)^{2}}{32 \eta(\eta+1)(2 \eta+1)^{2}} .
$$

By deriving the first and second order conditions of $\Pi_{\mathrm{R} 2}^{\mathrm{UU}^{*}}-\Pi_{\mathrm{R} 2}^{\mathrm{UP}}$, we have:

$$
\begin{aligned}
& \frac{\partial\left(\Pi_{\mathrm{R} 2}^{\mathrm{UU}^{*}}-\Pi_{\mathrm{R} 2}^{\mathrm{UP}}\right)}{\partial c_{m b}}=\frac{49\left(4 \eta^{2}+5 \eta+1\right) c_{m a}+\left(64 \eta^{2}-66 \eta-49\right) c_{m b}-2 \eta\left(32 \eta^{2}+97 \eta+65\right)}{784 \eta(\eta+1)(2 \eta+1)} \\
& \frac{\partial^{2}\left(\Pi_{\mathrm{R} 2}^{\mathrm{UU}}-\Pi_{\mathrm{R} 2}^{\mathrm{UP}}\right.}{\partial c_{m b}^{2}}=\frac{32 \eta-49}{784 \eta(\eta+1)} .
\end{aligned}
$$

Here we have three cases to consider, i.e., (1) $0<\eta<\frac{49}{32}$, (2) $\eta>\frac{49}{32}$ and (3) $\eta=\frac{49}{32}$.

(1) When $0<\eta<\frac{49}{32}$, we have $\frac{\partial^{2}\left(\Pi_{\mathrm{R} 2}^{\mathrm{UU}}-\Pi_{\mathrm{R} 2}^{\mathrm{UP}}\right)}{\partial c_{m b}^{2}}<0$, which tells that $\Pi_{\mathrm{R} 2}^{\mathrm{UU}^{*}}-\Pi_{\mathrm{R} 2}^{\mathrm{UP}}{ }^{*}$ is concave on $c_{m b}$. By solving $\Pi_{\mathrm{R} 2}^{\mathrm{UU}^{*}}-\Pi_{\mathrm{R} 2}^{\mathrm{UP}}=0$, we have two roots:

$$
\begin{gathered}
\tilde{c}_{m b, 3}=\frac{(\eta+1)\left(2 \eta(32 \eta+65)-7(4 \eta+1)\left(7 c_{m a}-\frac{4 \sqrt{2}\left(c_{m a}-1\right)}{\sqrt{\frac{1}{\eta}}}\right)\right)}{(2 \eta+1)(32 \eta-49)} \\
\tilde{c}_{m b, 4}=\frac{(\eta+1)\left(7(4 \eta+1)\left(-\frac{4 \sqrt{2}\left(c_{m a}-1\right)}{\sqrt{\frac{1}{\eta}}}-7 c_{m a}\right)+2 \eta(32 \eta+65)\right)}{(2 \eta+1)(32 \eta-49)} .
\end{gathered}
$$

Because

$$
\tilde{c}_{m b, 3}-\tilde{c}_{m b, 4}=\frac{56 \sqrt{2} \eta \sqrt{\frac{\left(4 \eta^{2}+5 \eta+1\right)^{2}\left(c_{m a}-1\right)^{2}}{\eta(2 \eta+1)^{2}}}}{49-32 \eta}
$$

which implies that $\tilde{c}_{m b, 3}-\tilde{c}_{m b, 4}>0$ since $0<\eta<\frac{49}{32}$. Note that we are working under the constraint that demands are positive which requires that $c_{m b}>\frac{(\eta+1)\left((4 \eta+1) c_{m a}-2 \eta\right)}{2 \eta+1}=\bar{c}_{m b, 3}$ and $c_{m b}<$ $\min \left\{\frac{(\eta+1)\left(c_{m a}+2 \eta\right)}{2 \eta+1}, \eta+1\right\}=\frac{(\eta+1)\left(c_{m a}+2 \eta\right)}{2 \eta+1}=\bar{c}_{m b, 4}$. To find the location of the key thresholds, we first compare $\tilde{c}_{m b, 3}$ with $\bar{c}_{m b, 4}$, which gives that

$$
\tilde{c}_{m b, 3}-\bar{c}_{m b, 4}=\frac{4\left(7 \sqrt{2} \sqrt{\frac{1}{\eta}}+\frac{28 \sqrt{2}}{\sqrt{\frac{1}{\eta}}}-57\right) \eta(\eta+1)\left(c_{m a}-1\right)}{(2 \eta+1)(32 \eta-49)} .
$$

It could be derived that $\tilde{c}_{m b, 3}-\bar{c}_{m b, 4}>0$ when $0<\eta<\frac{2}{49}$ and $\tilde{c}_{m b, 3}-\bar{c}_{m b, 4}<0$ when $\frac{2}{49}<\eta<\frac{49}{32}$. Secondly, by comparing $\tilde{c}_{m b, 4}$ with $\bar{c}_{m b, 3}$, which gives that

$$
\tilde{c}_{m b, 4}-\bar{c}_{m b, 3}=-\frac{4\left(7 \sqrt{2} \sqrt{\frac{1}{\eta}}+8\right) \eta(\eta+1)(4 \eta+1)\left(c_{m a}-1\right)}{(2 \eta+1)(32 \eta-49)}
$$

which is negative since $0<\eta<\frac{49}{32}$. Thus, in this case, we can conclude that there exist two subcases: When $0<\eta<\frac{2}{49}, \Pi_{\mathrm{R} 2}^{\mathrm{UU}}-\Pi_{\mathrm{R} 2}^{\mathrm{UP}}{ }^{*}$ is positive if $\bar{c}_{m b, 3}<c_{m b}<\bar{c}_{m b, 4}$ and $\Pi_{\mathrm{R} 2}^{\mathrm{UU}^{*}}-\Pi_{\mathrm{R} 2}^{\mathrm{UP}^{*}}$ is negative if $\bar{c}_{m b, 4}<c_{m b}<\tilde{c}_{m b, 3}$; When $\frac{2}{49}<\eta<\frac{49}{32}, \Pi_{\mathrm{R} 2}^{\mathrm{UU}^{*}}-\Pi_{\mathrm{R} 2}^{\mathrm{UP}}$ is positive all the time. 
(2) When $\eta>\frac{49}{32}$, it is straightforward to have $\frac{\partial^{2}\left(\Pi_{\mathrm{R} 2}^{\mathrm{UU}}-\Pi_{\mathrm{R} 2}^{*}\right)}{\partial c_{m b}^{2}}>0$, which tells that $\Pi_{\mathrm{R} 2}^{\mathrm{UU}}{ }^{*}-\Pi_{\mathrm{R} 2}^{\mathrm{UP}}{ }^{*}$ is convex on $c_{m b}$. In this case we have $\tilde{c}_{m b, 3}-\tilde{c}_{m b, 4}<0$ and $\tilde{c}_{m b, 3}$ is the smaller root of $\Pi_{\mathrm{R} 2}^{\mathrm{UU}}{ }^{*}-\Pi_{\mathrm{R} 2}^{\mathrm{UP}}{ }^{*}=0$. By solving the axis of symmetry of $\Pi_{\mathrm{R} 2}^{\mathrm{UU}^{*}}-\Pi_{\mathrm{R} 2}^{\mathrm{UP}}$, we have $c_{m b}=\frac{(\eta+1)\left(2 \eta(32 \eta+65)-49(4 \eta+1) c_{m a}\right)}{(2 \eta+1)(32 \eta-49)}$. Because $\frac{(\eta+1)\left(2 \eta(32 \eta+65)-49(4 \eta+1) c_{m a}\right)}{(2 \eta+1)(32 \eta-49)}-\bar{c}_{m b, 4}=-\frac{228 \eta(\eta+1)\left(c_{m a}-1\right)}{(2 \eta+1)(32 \eta-49)}>0$ when $\eta>\frac{49}{32}$, thus, we do not have to consider the larger root $\left(\tilde{c}_{m b, 4}\right)$. Further, we have that when $\Pi_{\mathrm{R} 2}^{\mathrm{UU}^{*}}-\Pi_{\mathrm{R} 2}^{\mathrm{UP}}$ is positive if $\bar{c}_{m b, 3}<c_{m b}<\tilde{c}_{m b, 3}$, and $\Pi_{\mathrm{R} 2}^{\mathrm{UU}^{*}}-\Pi_{\mathrm{R} 2}^{\mathrm{UP}}$ is negative all the time if $\tilde{c}_{m b, 3}<c_{m b}<\bar{c}_{m b, 4}$.

(3) When $\eta=\frac{49}{32}, \Pi_{\mathrm{R} 2}^{\mathrm{UU}^{*}}-\Pi_{\mathrm{R} 2}^{\mathrm{UP}}$ is a linear decreasing function of $c_{m b}$. We have $\Pi_{\mathrm{R} 2}^{\mathrm{UU}^{*}}-\Pi_{\mathrm{R} 2}^{\mathrm{UP}}=0$ when $c_{m b}=\frac{81\left(57 c_{m a}+8\right)}{2080}$. Because $\bar{c}_{m b, 3}<\frac{81\left(57 c_{m a}+8\right)}{2080}<\bar{c}_{m b, 4}$, therefore it is straightforward to find that $\Pi_{\mathrm{R} 2}^{\mathrm{UU}^{*}}-\Pi_{\mathrm{R} 2}^{\mathrm{UP}^{*}}>0$ if $\bar{c}_{m b, 3}<c_{m b}<\frac{81\left(57 c_{m a}+8\right)}{2080}$ and $\Pi_{\mathrm{R} 2}^{\mathrm{UU}^{*}}-\Pi_{\mathrm{R} 2}^{\mathrm{UP}}{ }^{*}>0$ if $\frac{81\left(57 c_{m a}+8\right)}{2080}<c_{m b}<\bar{c}_{m b, 4}$.

Proof of Proposition 5.1. We have shown that in scenario PP when R1 is inactive to choose product $\mathrm{b}$ and choose to sell product a, R2 would choose to sell product $\mathrm{b}$ if and only if when $\eta>\frac{2}{49}$ and $\bar{c}_{m b, 1}<c_{m b}<\tilde{c}_{m b, 1}$ are satisfied simultaneously. By solving R1's profit difference in scenarios PU and PP, we have:

$$
\Pi_{\mathrm{R} 1}^{\mathrm{PU}^{*}}-\Pi_{\mathrm{R} 1}^{\mathrm{PP}^{*}}=\frac{\left(-2 \eta c_{m a}-c_{m a}+c_{m b}+\eta\right)^{2}}{32 \eta^{2}+16 \eta}-\frac{2}{49}\left(c_{m a}-1\right)^{2} .
$$

The first and second order conditions respectively give that:

$$
\begin{aligned}
\frac{\partial\left(\Pi_{\mathrm{R} 1}^{\mathrm{PU}}-\Pi_{\mathrm{R} 1}^{\mathrm{PP}^{*}}\right)}{\partial c_{m b}} & =\frac{2\left(\eta-2 \eta c_{m a}-c_{m a}+c_{m b}\right)}{32 \eta^{2}+16 \eta} \\
\frac{\partial^{2}\left(\Pi_{\mathrm{R} 1}^{\mathrm{PU}}-\Pi_{\mathrm{R} 1}^{\mathrm{PP}}\right)}{\partial c_{m b}^{2}} & =\frac{2}{32 \eta^{2}+16 \eta}>0 .
\end{aligned}
$$

By solving $\Pi_{\mathrm{R} 1}^{\mathrm{PU}^{*}}-\Pi_{\mathrm{R} 1}^{\mathrm{PP}}=0$, we have two roots as follows:

$$
\begin{aligned}
& \hat{c}_{m b, 1}=\frac{1}{7}\left(-(2 \eta+1)\left(4 \sqrt{2} \eta \sqrt{\frac{\left(c_{m a}-1\right)^{2}}{\eta(2 \eta+1)}}-7 c_{m a}\right)-7 \eta\right) \\
& \hat{c}_{m b, 2}=\frac{1}{7}\left((2 \eta+1)\left(4 \sqrt{2} \eta \sqrt{\frac{\left(c_{m a}-1\right)^{2}}{\eta(2 \eta+1)}}+7 c_{m a}\right)-7 \eta\right)
\end{aligned}
$$

with $\hat{c}_{m b, 1}<\hat{c}_{m b, 2}$. Because

$$
\begin{aligned}
\hat{c}_{m b, 1}-\bar{c}_{m b, 2} & =\frac{1}{7}\left(-(2 \eta+1)\left(-4 \sqrt{2} \eta \sqrt{\frac{1}{\eta(2 \eta+1)}}\left(c_{m a}-1\right)-7 c_{m a}\right)-7 \eta\right)-\left(2 \eta c_{m a}+c_{m a}-\eta\right) \\
& =\frac{4}{7} \sqrt{2} \eta(2 \eta+1) \sqrt{\frac{1}{2 \eta^{2}+\eta}}\left(c_{m a}-1\right)<0 .
\end{aligned}
$$

Because $\Pi_{\mathrm{R} 1}^{\mathrm{PU}}-\Pi_{\mathrm{R} 1}^{\mathrm{PP}}$ is convex on $c_{m b}$, which tells that $\Pi_{\mathrm{R} 1}^{\mathrm{PU}}-\Pi_{\mathrm{R} 1}^{\mathrm{PP}}$ is negative all the time in the interval $\bar{c}_{m b, 1}<c_{m b}<\bar{c}_{m b, 2}$, and thus we can derive that there is no Pareto improvement for both retailers R1 and R2.

Proof of Propositions 5.2 and 5.3. In scenario UP, when R1 adopts to sell product $\mathrm{b}$ and $\mathrm{R} 2$ does not, to survey retailer R1's profit change, we first derive the profit difference as:

$$
\Pi_{\mathrm{R} 1}^{\mathrm{UP}^{*}}-\Pi_{\mathrm{R} 1}^{\mathrm{PP}^{*}}=\frac{\left((\eta+1) c_{m a}-(2 \eta+1) c_{m b}+2 \eta(\eta+1)\right)^{2}}{16 \eta(\eta+1)(2 \eta+1)}-\frac{2}{49}\left(c_{m a}-1\right)^{2} .
$$


The first and second order conditions on $c_{m b}$ give that:

$$
\begin{aligned}
\frac{\partial\left(\Pi_{\mathrm{R} 1}^{\mathrm{UP}}-\Pi_{\mathrm{R} 1}^{\mathrm{PP}}\right)}{\partial c_{m b}} & =\frac{(2 \eta+1) c_{m b}-(\eta+1)\left(c_{m a}+2 \eta\right)}{8 \eta(\eta+1)} \\
\frac{\partial^{2}\left(\Pi_{\mathrm{R} 1}^{\mathrm{UP}}-\Pi_{\mathrm{R} 1}^{\mathrm{PP}}\right)}{\partial c_{m b}^{2}} & =\frac{2 \eta+1}{8 \eta^{2}+8 \eta}>0 .
\end{aligned}
$$

This tells that function $\Pi_{\mathrm{R} 1}^{\mathrm{UP}}-\Pi_{\mathrm{R} 1}^{\mathrm{PP}^{*}}$ is convex with two roots for $\Pi_{\mathrm{R} 1}^{\mathrm{UP}^{*}}-\Pi_{\mathrm{R} 1}^{\mathrm{PP}}=0$ :

$$
\begin{aligned}
\hat{c}_{m b, 3} & =-\frac{(\eta+1)\left(2 \eta\left(-2 \sqrt{\frac{4 \eta+2}{\eta^{2}+\eta}}\left(c_{m a}-1\right)-7\right)-7 c_{m a}\right)}{14 \eta+7} \\
\hat{c}_{m b, 4} & =\frac{(\eta+1)\left(2 \eta\left(7-2 \sqrt{\frac{4 \eta+2}{\eta^{2}+\eta}}\left(c_{m a}-1\right)\right)+7 c_{m a}\right)}{14 \eta+7}
\end{aligned}
$$

and

$$
\hat{c}_{m b, 3}-\hat{c}_{m b, 4}=\frac{8 \sqrt{2}\left(c_{m a}-1\right)}{7 \sqrt{\frac{1}{\eta+1}+\frac{1}{\eta}}}<0
$$

this implies that $\hat{c}_{m b, 3}<\hat{c}_{m b, 4}$. To study how R1's profit changes in scenario UP, we have to work under the premise that R2 would choose product a (there exist four conditions that are given in Prop. 4.4) and all the discussions on $c_{m b}$ must be located in the interval $\bar{c}_{m b, 3}<c_{m b}<\bar{c}_{m b, 4}$, which ensures the non-negativity of demands. To obtain accurate results, we have to narrow our search. By deriving:

$$
\begin{aligned}
\hat{c}_{m b, 4}-\bar{c}_{m b, 4} & =\frac{(\eta+1)\left(2 \eta\left(2 \sqrt{2} \sqrt{\frac{(2 \eta+1)\left(c_{m a}-1\right)^{2}}{\eta(\eta+1)}}+7\right)+7 c_{m a}\right)}{14 \eta+7}-\frac{(\eta+1)\left(c_{m a}+2 \eta\right)}{2 \eta+1} \\
& =\frac{4 \sqrt{2}\left(c_{m a}-1\right)^{2}}{7 \sqrt{\frac{(2 \eta+1)\left(c_{m a}-1\right)^{2}}{\eta(\eta+1)}}}>0
\end{aligned}
$$

this indicates that we do not need to consider $\hat{c}_{m b, 4}$ in some of the following proof. We work on the proof under the following three cases:

(1) When $0<\eta<\frac{49}{32}$, in this case, $\Pi_{\mathrm{R} 2}^{\mathrm{UP}}-\Pi_{\mathrm{R} 2}^{\mathrm{UU}^{*}}$ and $\Pi_{\mathrm{R} 1}^{\mathrm{UP}}-\Pi_{\mathrm{R} 1}^{\mathrm{PP}}$ are both convex function of $c_{m b}$. Because

$$
\begin{aligned}
\hat{c}_{m b, 3}-\bar{c}_{m b, 4} & =-\frac{(\eta+1)\left(c_{m a}+2 \eta\right)}{2 \eta+1}-\frac{(\eta+1)\left(2 \eta\left(-2 \sqrt{2} \sqrt{\frac{2 \eta+1}{\eta(\eta+1)}}\left(c_{m a}-1\right)-7\right)-7 c_{m a}\right)}{14 \eta+7} \\
& =\frac{4 \sqrt{2}\left(c_{m a}-1\right)}{7 \sqrt{\frac{1}{\eta+1}+\frac{1}{\eta}}}<0 .
\end{aligned}
$$

Then, we could conclude that when $0<\eta<\frac{49}{32}$, no matter $\hat{c}_{m b, 3} \leq \bar{c}_{m b, 3}$ or $\hat{c}_{m b, 3}>\bar{c}_{m b, 3}$, the co-existence of $\Pi_{\mathrm{R} 2}^{\mathrm{UP}^{*}}>\Pi_{\mathrm{R} 2}^{\mathrm{UU}}$ and $\Pi_{\mathrm{R} 1}^{\mathrm{UP}}>\Pi_{\mathrm{R} 1}^{\mathrm{PP}}$ could not hold at the same time. See Figure B.1 as below.

(2) When $\eta>\frac{49}{32}$, it could be found that $\Pi_{\mathrm{R} 2}^{\mathrm{UP}^{*}}-\Pi_{\mathrm{R} 2}^{\mathrm{UU}^{*}}$ and $\Pi_{\mathrm{R} 1}^{\mathrm{UP}}-\Pi_{\mathrm{R} 1}^{\mathrm{PP}^{*}}$ are concave and convex function (of $\left.c_{m b}\right)$ respectively. Because

$$
\hat{c}_{m b, 3}-\bar{c}_{m b, 3}=\frac{(\eta+1)\left((4 \eta+1) c_{m a}-2 \eta\right)}{2 \eta+1}-\frac{(\eta+1)\left(2 \eta\left(-2 \sqrt{2} \sqrt{\frac{2 \eta+1}{\eta(\eta+1)}}\left(c_{m a}-1\right)-7\right)-7 c_{m a}\right)}{14 \eta+7}
$$




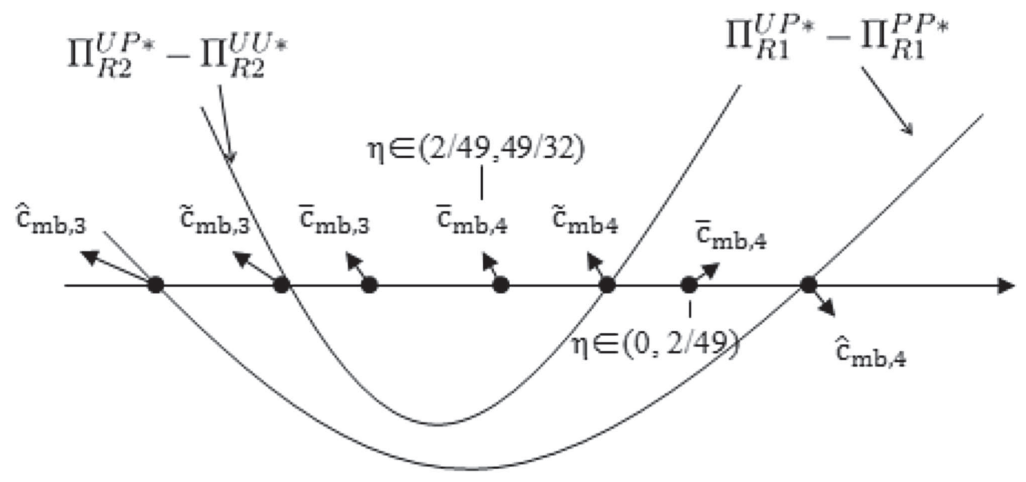

FiguRE B.1. Retailers R1 and R2's profits in scenario UP when $0<\eta<\frac{49}{32}$.

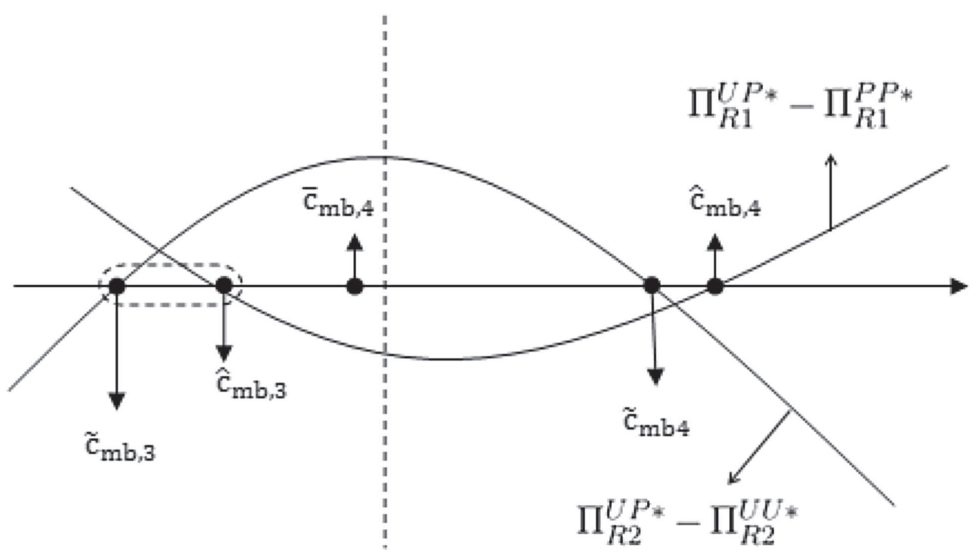

Figure B.2. Retailers R1 and R2's profits in scenario UP when $\eta>\frac{49}{32}$.

$$
=\frac{4 \eta(\eta+1)\left(\sqrt{\frac{4 \eta+2}{\eta^{2}+\eta}}-7\right)\left(c_{m a}-1\right)}{14 \eta+7} .
$$

Then, we could conclude that if and only if $\hat{c}_{m b, 3}>\bar{c}_{m b, 3}$ (or equivalently if $\eta>\frac{1}{98}(\sqrt{2417}-45)$ ) is satisfied then the co-existence of $\Pi_{\mathrm{R} 2}^{\mathrm{UP}}>\Pi_{\mathrm{R} 2}^{\mathrm{UU}}$ and $\Pi_{\mathrm{R} 1}^{\mathrm{UP}}>\Pi_{\mathrm{R} 1}^{\mathrm{PP}}$ holds. One could find that if $0<\eta \leq$ $\frac{1}{98}(\sqrt{2417}-45)\left(\hat{c}_{m b, 3} \leq \bar{c}_{m b, 3}\right)$, no Pareto improvement exists. See Figure B.2.

(3) When $\eta=\frac{49}{32}$, it could be found that $\Pi_{\mathrm{R} 2}^{\mathrm{UP}}{ }^{*}-\Pi_{\mathrm{R} 2}^{\mathrm{UU}}{ }^{*}$ and $\Pi_{\mathrm{R} 1}^{\mathrm{UP}}{ }^{*}-\Pi_{\mathrm{R} 1}^{\mathrm{PP}^{*}}$ are concave and convex function (of $c_{m b}$ ) respectively. The rest of the proof is similar to cases (2) and (3), and we choose to omit it.

Acknowledgements. The work was supported by the Philosophy and Social Science Project of Anhui Province [AHSKY2016D25].

\section{REFERENCES}

[1] M. Agi and X. Yan, Greening products in a supply chain under market segmentation and different channel power structures. Int. J. Prod. Econ. 223 (2020) 107523. 
[2] S.M. Ali, M.H. Rahman, T.J. Tumpa, A.A.M. Rifat and S.K. Paul, Examining price and service competition among retailers in a supply chain under potential demand disruption. J. Retail. Consum. Serv. 40 (2018) 40-47.

[3] G. Aust and U. Buscher, Game theoretic analysis of pricing and vertical cooperative advertising of a retailer-duopoly with a common manufacturer. Cent. Eur. J. Oper. Res. 24 (2016) 127-147.

[4] D. Baum, M. Spann, J. Fuller and C. Thurridl, The impact of social media campaigns on the success of new product introductions. J. Retail. Consum. Serv. 50 (2019) 289-297.

[5] B.L. Bayus, G. Erickson and R. Jacobson, The financial rewards of new product introductions in the personal computer industry. Manag. Sci. 49 (2003) 197-210.

[6] O. Bianchi, Why do some new services or products fail. Mark. Challenge 7 (2004) 1-3.

[7] E. Brynjolfsson, Y. Hu and M.S. Rahman, Battle of the retail channels: how product selection and geography drive cross-channel competition. Manag. Sci. 55 (2009) 1755-1765.

[8] S. Choi and K. Fredj, Price competition and store competition: store brands vs. national brand. Eur. J. Oper. Res. 225 (2013) 166-178.

[9] E. Dahan, A. Soukhoroukova and M. Spann, New product development 2.0: preference markets - how scalable securities markets identify winning product concepts and attributes. J. Prod. Innovation Manag. 27 (2010) 937-954.

[10] R. Desiraju, New product introductions, slotting allowances, and retailer discretion. J. Retail. 77 (2001) 335-358.

[11] C. Dong, Q. Liu and B. Shen, To be or not to be green? Strategic investment for green product development in a supply chain. Transp. Res. Part E: Logist. Transp. Rev. 131 (2019) 193-227.

[12] M.A. Dulebenets, M.M. Golias and S. Mishra, The green vessel schedule design problem: consideration of emissions constraints. Energy Syst. 8 (2017) 761-783.

[13] T. Düsterhöft, A. Hübner and K. Schaal, A practical approach to the shelf-space allocation and replenishment problem with heterogeneously sized shelves. Eur. J. Oper. Res. 282 (2020) 252-266.

[14] T. Ertay and S.I. Satoğlu, System parameter selection with information axiom for the new product introduction to the hybrid manufacturing systems under dual-resource constraint. Int. J. Prod. Res. 50 (2012) 1825-1839.

[15] Y. Fang and B. Shou, Managing supply uncertainty under supply chain Cournot competition. Eur. J. Oper. Res. 243 (2015) $156-176$.

[16] M. Farhat, A. Akbalik, A.B. Hadj-Alouane and N. Sauer, Lot sizing problem with batch ordering under periodic buyback contract and lost sales. Int. J. Prod. Econ. 208 (2019) 500-511.

[17] A. Hafezalkotob, Competition, cooperation, and coopetition of green supply chains under regulations on energy saving levels. Transp. Res. Part E: Logist. Transp. Rev. 97 (2017) 228-250.

[18] M.A. Khan and T. Wuest, Upgradable product-service systems: implications for business model components. Procedia CIRP 80 (2019) 768-773.

[19] Z.L. Liu, T.D. Anderson and J.M. Cruz, Consumer environmental awareness and competition in two-stage supply chains. Eur. J. Oper. Res. 218 (2012) 602-613.

[20] I. Lobel, J. Patel, G. Vulcano and J. Zhang, Optimizing product launches in the presence of strategic consumers. Manag. Sci. 62 (2015) 1778-1799.

[21] L. Luo, P.K. Kannan and B.T. Ratchford, New product development under channel acceptance. Mark. Sci. 26 (2007) 149-163.

[22] A. Mehra, R. Bala and R. Sankaranarayanan, Competitive behavior-based price discrimination for software upgrades. Inf. Syst. Res. 23 (2012) 60-74.

[23] D. Nuscheler, A. Engelen and S.A. Zahra, The role of top management teams in transforming technology-based new ventures' product introductions into growth. J. Bus. Venturing 34 (2019) 122-140.

[24] B. Pal and A. Sarkar, Optimal strategies of a dual-channel green supply chain with recycling under retailer promotional effort. RAIRO: OR $\mathbf{5 5}$ (2021) 415-431.

[25] M. Palmer and Y. Truong, The impact of technological green new product introductions on firm profitability. Ecol. Econ. 136 (2017) 86-93.

[26] C. Pan, Manufacturer's direct distribution with incumbent retailer's product line choice. Econ. Lett. 174 (2019) $136-139$.

[27] S. Parsaeifar, A. Bozorgi-Amiri, A. Naimi-Sadigh and M.S. Sangari, A game theoretical for coordination of pricing, recycling, and green product decisions in the supply chain. J. Clean. Prod. 226 (2019) 37-49.

[28] O. Perdikaki, D. Kostamis and J.M. Swaminathan, Timing of service investments for retailers under competition and demand uncertainty. Eur. J. Oper. Res. 254 (2016) 188-201.

[29] M. Reisi, S.A. Gabriel and B. Fahimnia, Supply chain competition on shelf space and pricing for soft drinks: a bilevel optimization approach. Int. J. Prod. Econ. 211 (2019) 237-250.

[30] J.W. Ridge, S. Johnson, A.D. Hill and J. Bolton, The role of top management team attention in new product introductions. J. Bus. Res. 70 (2017) 17-24.

[31] P. Sok and A. O'Cass, Examining the new product innovation-performance relationship: optimizing the role of individual-level creativity and attention-to-detail. Ind. Mark. Manag. 47 (2015) 156-165.

[32] A.B. Sorescu and J. Spanjol, Innovation's effect on firm value and risk: insights from consumer packaged goods. J. Mark. 72 (2008) 114-132.

[33] C.F. Wang, L.Y. Chen and S.C. Chang, International diversification and the market value of new product introduction. J. Int. Manag. 17 (2011) 333-347.

[34] B. Yalabik and R.J. Fairchild, Customer, regulatory, and competitive pressure as drivers of environmental innovation. Int. J. Prod. Econ. 131 (2011) 519-527. 
[35] S. Yang, C.V. Shi, Y. Zhang and J. Zhu, Price competition for retailers with profit and revenue targets. Int. J. Prod. Econ. 154 (2014) 233-242.

[36] A. Yenipazarli and A. Vakharia, Pricing, market coverage and capacity: can green and brown products co-exist? Eur. J. Oper. Res. 242 (2015) 304-315.

[37] R. Yin, H. Li and C.S. Tang, Optimal pricing of two successive-generation products with trade-in options under uncertainty. Decis. Sci. 46 (2015) 565-595.

[38] X. Yu, Y. Lan and R. Zhao, Strategic green technology innovation in a two-stage alliance: vertical collaboration or codevelopment? Omega 98 (2021) 102116.

[39] Q. Zhang, Q.H. Zhao, X. Zhao and L. Tang, On the introduction of green product to a market with environmentally conscious consumers. Comput. Ind. Eng. 139 (2020) 106190.

[40] W. Zhu and Y. He, Green product design in supply chains under competition. Eur. J. Oper. Res. 258 (2017) 165-180. 